\title{
A Remarkable Recent Transition in the Solar Dynamo
}

\author{
C. de Jager ${ }^{1}$ (D) S.-I. Akasofu' ${ }^{2}$ S. Duhau ${ }^{3}$. \\ W.C. Livingston ${ }^{4} \cdot$ H. Nieuwenhuijzen ${ }^{5}$ - M.S. Potgieter ${ }^{6}$
}

Received: 6 December 2015 / Accepted: 20 September 2016 / Published online: 20 October 2016

(C) The Author(s) 2016. This article is published with open access at Springerlink.com

\begin{abstract}
We summarize the major aspects of the remarkable, fairly long lasting period ( $\sim 2005$ to $\sim 2010$ ) of low solar activity, that we will call the Transition. It is the transitional stage between the Grand Maximum of the 20th century and a forthcoming (most probably Regular) episode of solar activity. The various kinds of activity in the functioning of the equatorial components of the solar dynamo before and during the Transition are summarized. While the behavior of unipolar magnetic regions and their rest-latitudes already gave very early indications - mid 20th century - of the forthcoming Transition, more such indications became available around 1995 and the main part of it occurred between 2005 and 2010. Some of the inferences are discussed. We submit the hypothesis that the solar tachocline undergoes pulsations and we present some helioseismic evidences. In that scenario we find that its equatorial part has moved downward over a fairly small semi-amplitude $(\sim 0.03$ solar radii) during the time of the Transition. There are several indications, apart from this 'pulsation', that the tachocline may even be pulsating with still smaller amplitudes in more modes. We speculate about the physical mechanism(s).
\end{abstract}

Keywords Equatorial magnetic fields · Polar magnetic fields · Grand maximum · Transition phase $\cdot$ Solar dynamo $\cdot$ Tachocline

C. de Jager worked formerly at SRON Laboratory for Space Research, Utrecht, The Netherlands

C. de Jager

info@cdejager.com

1 R. Netherlands Inst. Sea Research, Texel, The Netherlands

2 International Arctic Research Center (IARC), Univ. of Alaska, Fairbanks, USA

3 Departamento de Fisica, Facultad de Ingenieria, Univ. de Buenos Aires, Buenos Aires, Argentina

4 NOAO/National Solar Observatory, Tucson, AZ 85726-6732, USA

5 SRON Laboratory for Space Research, Utrecht, The Netherlands

6 Centre for Space Research, North-West Univ., Potchefstroom, South Africa 


\section{Introduction}

When, around the year 2005, the past Schwabe cycle (\#23) in solar activity had come down to its minimum, the expected rise to the maximum of cycle \#24 appeared to be considerably delayed. Contrary to initial expectations, the usual solar activity, such as the Total Solar Irradiation (TSI), sunspots, Coronal Mass Ejections, flares ... only started to increase in intensity, number and degree of activity in the period between 2008 and 2010.

This relatively long-lasting minimum had several unusual aspects. Earlier, Duhau and de Jager (2008) distinguished three kinds of relatively large episodes of solar activity, each of them containing about a dozen or more Schwabe cycles, viz. Grand Maxima, Grand Minima and episodes of Regular variations. Usoskin et al. (2014), who studied solar activity over a period of three millennia, confirmed this subdivision. The reality of the recent (20th century) Grand Maximum is confirmed in critical studies by Solanki et al. (2004), Lockwood et al. (2009, 2010), Lockwood (2012) and Clette et al. (2014), although, after the introduction of the new system of sunspot counting (Clette et al. 2014; Svalgaard and Schatten 2016), the maximum is less extreme than was thought before. Clette et al. (2014) call it the 'Modern Maximum'. In order to avoid confusion we will continue to call it the '20th century Grand Maximum' or briefly the 'Grand Maximum'.

The Grand Maximum started in 1924 (Duhau and Chen 2002; Duhau and de Jager 2010) while Lockwood et al. (2009) place the beginning in 1920. For the end of it Duhau and de Jager (2010) find 2008 (cf. our Table 1 later in this paper, and the corresponding text), while Lockwood et al. (2009), who studied the open solar flux, found, from linear extrapolations, terminal years of 2014 (for the open solar flux), 2027 (from interplanetary magnetic field data) and 2013 (solar wind velocity).

The period that we are discussing here is the time interval between the past Grand Maximum and a forthcoming Episode of which Duhau and de Jager (2010) and de Jager and Duhau (2012) give arguments that it will be of the Regular type. In this paper we will call this transitional period the Transition. Feynman and Ruzmaikin (2014) give examples of more such transitions that may have existed in the past.

Other authors have already mentioned this Transition. We refer to papers and reviews by Russell et al. (2010), Lockwood et al. (2010), Lockwood (2012), Nandy et al. (2011), Solanki and Krivova (2011), de Jager and Duhau (2011), de Jager (2012). Quoting Russell et al. (2010): "Previous solar minima had occurred in 1996, 1986, 1976 and 1966, so the latest cycle had been expected to reach its minimum in 2006, but in 2007 the sunspot number and every other indicator of solar activity continued to drop, as they did through 2008 ...". Russell et al.'s paper was written mid-2009 and we now know that the extended minimum continued till 2010.

Uncertainty remained at that time, as appears from a quotation by Weiss (2010): Is this just an abnormal fluctuation or are we about to experience a Maunder-like Grand Minimum? The answer can be given, based on a look a the sunspot curve. This recent extended minimum is the longest of the past century. More of them have occurred earlier in the past (some of them listed by Feynman and Ruzmaikin 2014). Examples of sunspot cycles with extreme minima are cycle \#13 (maximum in 1894; minimum sunspot number 1.7), and cycle \#14 (maximum in 1906; minimum sunspot number 1.5). We compare these with the last fully observed cycle \#23: its minimum sunspot number was 1.7 - just comparable. We note that these sunspot numbers are given according to the 'classical' counting system - cf. the remark at the end of this section. The difference between these extreme minima and the present Transition phase is that this last one is the first one that could be observed by modern observational techniques. 
This period of solar relative inactivity had several aspects: these are partly related to the polar and partly to the equatorial part of solar activity, the two main solar magnetic regions. In this paper we first summarize and analyse existing observations. Thereafter these observations are synthesized into a scenario that links them to the two magnetically active solar regions and we examine the implications for our current understanding of part of the functioning of the solar dynamo. We will forward a new suggestion, viz. that the solar tachocline is pulsating. Quantitatively, we find that these 'pulsations' need to have a remarkably small amplitude in order to explain the observations. Finally, we present some reflections on the possible mechanism.

Remark on Sunspot Numbers and Numbering System Around the beginning of this century the Zürich sunspot numbering system introduced by Wolf and developed further by Waldmeier and successors (The Wolf Sunspot Numbers, generally called International Sunspot Numbers, ISN) was replaced by a new system of sunspot numbering (the Modified International Sunspot Numbers (MISN; Clette et al. 2014), while another new counting system is called the Group Sunspot Numbers (GSN; Hoyt and Schatten 1998; Hathaway et al. 2002; Svalgaard and Schatten 2016). The main reason for the change to the MISN was to overcome the 'Waldmeier discontinuity' (also described by Aparicio et al. 2012), while the introduction of the Group Sunspot Numbers was bases on the - likely - assumption that sunspot groups represent solar equatorial magnetic variability better than the Zürich system.

After these modifications it is inevitable that in this paper - which has partly the character of a review paper - diagrams or formulas appear that are based on one of the different systems of sunspot numbering. We realize that this is a confusing situation and try, in each particular case, to inform the reader about the system of numbering that we are dealing with.

\section{The Role of the Polar and Equatorial Magnetic Regions in Controlling Solar Activity Variations; Proxies for the Main Variables}

The fact that the solar surface presents two magnetically active regions is often overlooked, particularly by climatologists who study sun-climate relationships. In their research they nearly invariably restrict themselves to searching for the relation between climate and the magnetic activity in the sunspot region, where the latter is characterized by the number of sunspots on the disk. But the situation that the polar and equatorial areas of the sun are both dominated by magnetic fields and structures with approximately equally strong total magnetic fluxes is already known since the time that Babcock and Babcock (1955) introduced technical innovations in their spectral observations of magnetic fields that permitted them to observe fields as small as 0.5 Gauss. Babcock and Livingston (1958) studied the polar magnetic fields. According to their measurements the northern field had at that time an average strength of $0.2 \mathrm{G}$, while the southern average field strength was $0.6 \mathrm{G}$. They found that, on the average, the time of maximum of the polar field was delayed by 3 years after sunspot minimum.

Earlier work by Grotrian and Künzel (1950) had already given values for the total magnetic flux of the equatorial (sunspot) region and somewhat later work (review by de Jager 1959, p. 340 and following) showed that the polar and equatorial fluxes are approximately comparable in magnitude. The same result was obtained later by Makarov et al. (2004): the total flux from the 'large unipolar fields' (of order of a few gauss) is according to them by about a factor 2 larger than the flux from the polar faculae (about a few hundred Gauss each) and the latter is about a factor 2 larger than the total flux due to the sunspots. However, for 
the corresponding local magnetic energies the order is the opposite. In connection to this, we should add that the main issue is not how much flux the poles have in comparison with active lower latitudes, but the fact that they seem to be the clear precursors of the subsequent Schwabe cycle.

In view of the comparable total fluxes of the polar and equatorial magnetic fields it is necessary to consider both elements in discussions of solar variability. The role of the large unipolar fields is another item that has, so far, hardly been studied (cf. Sect. 6 of this paper).

The polar regions have only been subject of regular observations during the past half century and for a study of the long-term variation of polar activity the introduction of a proxy is necessary. The same applies to the equatorial region. The magnetic fields of the sunspots have been measured since the early part of the 20th century but not systematically so. Systematic observations of the facular magnetic field strengths around spots are known only since the 2 nd part of that century. Therefore, we need proxies in order to describe the time dependence of these two variables.

The sunspot number is related to the magnetic flux contained in the (equatorial) sunspot belt. That activity shows itself in different ways: sunspots, faculae, flares, Coronal Mass Ejections, just to mention the most important components. Since that ensemble of activities is only well known since the 2 nd half of the 20th century, its values are usually simulated by either the International Sunspot Number or the Group Sunspot Number. Both are generally taken as suitable proxies. A better proxy for the total magnetic energy contained in active regions would have been the integrated magnetic flux of all spots present, but the latter quantity has only been measured during part of the 20th century. Therefore, in the absence of a better proxy for the long-term total equatorial activity, one generally chooses $R_{\max }$, the maximum international sunspot numbers, or else, the maximum value of the recently introduced Group Number for subsequent Schwabe cycles. The $R_{\max }$ data are known from 1610 onward.

A direct measure for the total polar activity would be the sum of the magnetic fields of the polar faculae. There have been measured since the beginning of the 20th century (Sheeley 1966, 1976, 1991, 2008) and their calibrated values over the past 100 years are clearly correlated with the heliospheric magnetic fields. The measured values of the polar faculae are also in good agreement with both the polar fields and the polar flux estimates. This makes these data excellent proxies for studying the polar magnetic fields (MuñozJaramillo et al. 2012, 2013a, 2013b). A drawback is that data of the polar facular flux are only known since the beginning of the 20th century.

An alternative proxy for the total polar magnetic field strength is therefore the commonly used value of $a a_{\min }$, the minimum value of the geomagnetic $a a$ index for subsequent Schwabe cycles (Russell 1975; Russell and Mulligan 1995; Hathaway et al. 1999; Duhau and Chen 2002) The $a a_{\min }$ data are known from 1870 onward (Mayaud 1972) and the series has been extended down to 1844 by Nevalinna and Kataja (1993). The $a a_{\text {min }}$ data from 1856 onward were homogenized by M. Lockwood (priv. comm.) and published by de Jager and Duhau (2012). Still earlier $a a_{\min }$ data (before 1844) do exist in the literature (cf. Nagovitsyn 2005) but these are based on an assumed correlation between $R_{\max }$ and $a a_{\text {min }}$, while an essential part of this paper is that such a correlation should not be assumed and to the best has to be studied. Therefore such extrapolated data will not be used in this paper. (An exception is made for Figs. 12a and part of 12b, but we have to realize that for these diagrams the parts from before 1844 are unreliable.)

The fact that $a a_{\min }$ is a suitable proxy for the polar magnetic fields is also shown by diagrams such as Fig. 1 which presents the relation between $a a_{\min }$ and the total polar magnetic flux $D M_{\max }$. The correlation between these two quantities $\left(a a_{\min }\right.$ and polar flux $)$ is excellent. 
Fig. 1 The relation between

$a a_{\min }$ and the total polar magnetic flux $D M_{\max }$. The $a a_{\min }$ data are homogenized by M. Lockwood from 1856 onward and published by de Jager and Duhau (2012)

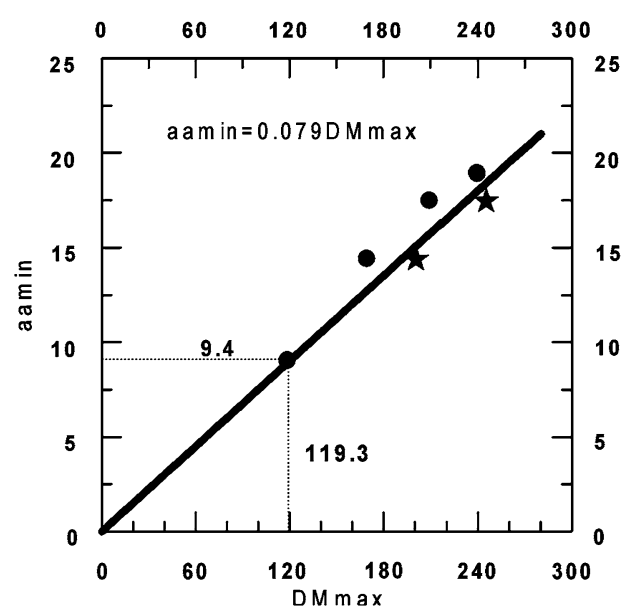

The $a a$ index as such carries information on both the toroidal and the poloidal components of the magnetic field. The first is related to the (equatorial) activity centers, the latter rather to the corotating interaction regions in the solar wind. When the $a a$ index reaches its minimum value the former contribution is very small. For that reason the use of the $a a_{\text {min }}$ proxy is advocated.

\section{The Main Aspects of Solar Activity During the Transition}

Turning to the theme of this paper, the Transition of 2005-2010, we reproduce as Fig. 2a a diagram due to Lockwood, published as an addendum to a review by de Jager (2012) after a study week at the Berne International Space Science Institute (Bengtsson et al. 2012). It shows nine different aspects of solar variability. Arrows point to the time of minimum or maximum, where the maxima refer to those variables that reach their maximum values at times of minimum solar activity, e.g. cosmic ray maximum appears at times of minimal solar wind flux.

As appears from the text in the frame of the diagram there are, roughly, two periods of solar extreme activity (in the sense as described above) during the period of the Transition. One of them is between 2008.5 and 2009.5 and a second one is between 2009.5 and 2010.5. The first time of extreme values is reached by the sunspot number, the middle UV spectral irradiance (SSI) and the MgII spectral lines in the near UV part of the spectrum. These variables are all directly related to the activity regions in the sunspot belt and to their medium-energy radiation fluxes. They are, hence, in one way or the other related to the Total Solar Irradiance (TSI), and in turn to the sunspot number $R$.

The late group of extreme values, those around 2010, is mainly related to the corpuscular solar emission of which the open solar flux (Lockwood et al. 2009; Lockwood 2013) is an important component. The magnetized part of the corpuscular radiation is related to the cosmic ray flux (cf. Sect. 5), because the latter is modulated by the interplanetary magnetized solar plasma. Maximum magnetized solar plasma corresponds to minimum cosmic ray flux and reversely so.

We compare these observations with those of the X-ray emitting jets emanating from coronal holes (Savcheva et al. 2009), while realizing that the coronal holes are chiefly related to the polar fields. The jets show a motion transverse to the jet outflow direction. This 
motion reflects the magnetic polarity orientation of the ephemeral active region at the base of the jets. The direction of the motion changed between 2006-2007 and 2008-2009. Remarkably enough, the preferred motion in the first period reflected the active regions of the coming sunspot cycle (\#24) while those of the latter period reflected the cycle \#25. These observations confirm that new cycles of solar activity start at high latitudes.

The data from Fig. 2a find further support in Fig. 2b, which shows the time variation of several variables that are strongly associated with the sunspot number, hence with the equatorial part of solar activity. The data from this latter diagram have been derived from several sources, that are listed in a diagram by Balogh et al. (2014). They show that all these sunspot-associated variables reach their minimum values in 2008.5-2009.0 with an uncertainty of \pm 0.5 year.

To further illustrate the variations relative to the sunspot belt activity we reproduce, as Fig. 3, a diagram due to Yeo et al. (2014). It gives an overview of the variation of the TSI from 1972 through 2011, hence including the years of the Transition. Among other things it also shows the increased accuracy of measurements with time. In the earlier part of the measurements, around 1986 the spreading is large, e.g. the ACRIM data being lower than all others, while in 2009 they are all nearly equal. The average value of the four curves confirms Lockwood's time of minimum TSI and Fig. 3 also shows that the (average of the) minimum TSI value of 2008.5 is the lowest of the three cycles shown. The difference is insignificantly small: it falls within the error bounds. Hence, this information cannot be used for the present study but it seems worth to keep it in mind for later investigations (Yeo et al. 2015). Anyway, realizing that the TSI reflects the concentration of magnetic elements in the chromosphere and specifically the magnetic flux of the facular fields around the sunspots, it shows that during the Transition the magnetic activity in the sunspot belt was very low.

De Jager et al. (2010) and de Jager and Nieuwenhuijzen (2013) have found that $34 \%$ or $24 \%$ respectively, of the sun-related long-term average terrestrial ground temperature variations (as measured from 1610 till 1950 and till 1912, respectively) are proportional to variations of the polar magnetic flux. They suggested that these latter variations are linked to the variations in the solar corpuscular flux, most probably the flux emanating from the coronal holes. It is significant that during the century before the year 2000 the average interplanetary magnetic field had doubled (Solanki et al. 2000) but that the solar wind velocity decreased steadily since about 2000. It reached a minimum value after 2009 (Lockwood et al. 2009; reproduced as Fig. 11 by Russell et al. 2010). This is one of the bases of part of the conclusions summarized in our Figs. $2 \mathrm{a}$ and $2 \mathrm{~b}$.

This particle flux, being a major part of the open solar flux (cf. Lockwood 2013) is most active at times near sunspot minimum and is associated with the polar magnetic regions and particularly with the coronal holes. These, in turn, are related to the poloidal field of the solar dynamo. Therefore, these observations confirm the above suggestion that the late ( 2010) minimum activity should be associated with the poloidal (polar) part of the sun's magnetic field while, as we noted before, the earlier TSI minimum (of 2008.5) is related to the equatorial (toroidal) part.

In conclusion, the extreme values of solar activity variation in the early part of the Transition $(\sim 2008.5)$ are related to variations in the equatorial magnetic fluxes, while those that regulate the later part $(\sim 2009-2010)$ are connected to that in the polar magnetic fields.

\section{Umbral Magnetic Fields}

A prominent aspect of solar variability during and partly preceding the Transition is an observed relatively stronger variation of the average maximum sunspot umbral field strength 


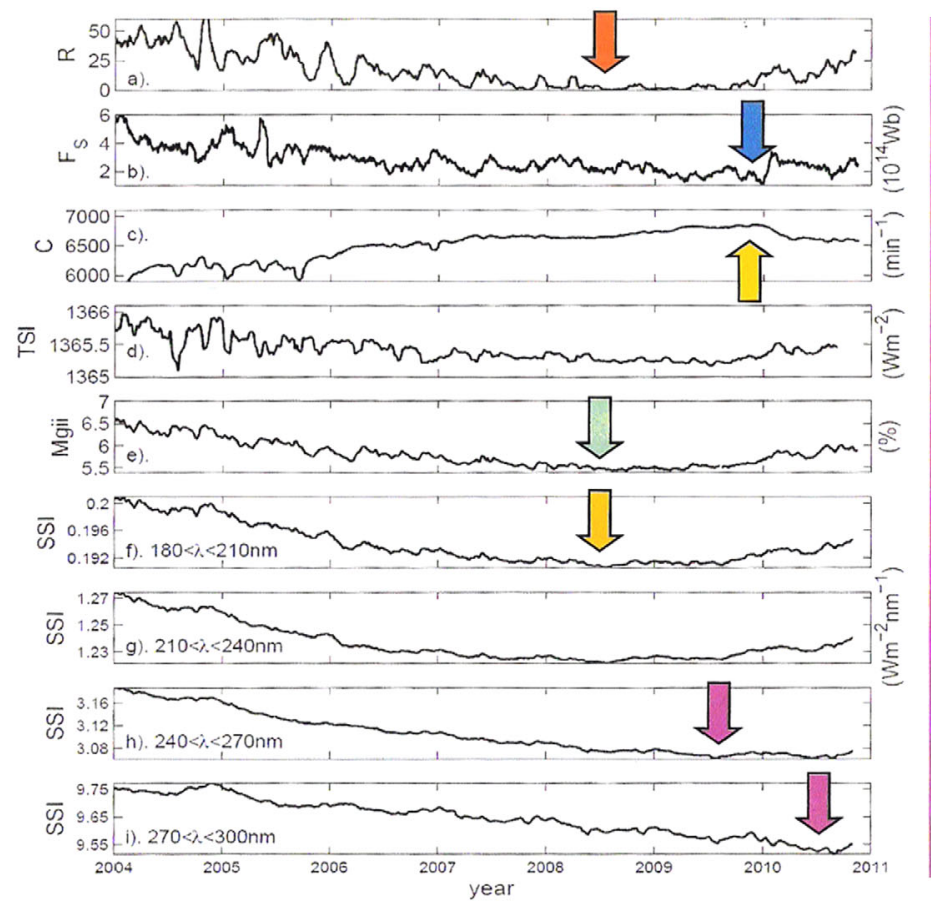

FUV $\square$ minimises

at around the

same time as

sunspot number,

$\mathrm{R}, \square$ and the Mgii

UV proxy $\square$

\section{Stratospheric-} effective MUV $\square$ declines more and longer - like open solar flux $\square$ (note when GCRs fluxes peak $\square$ )

(a)

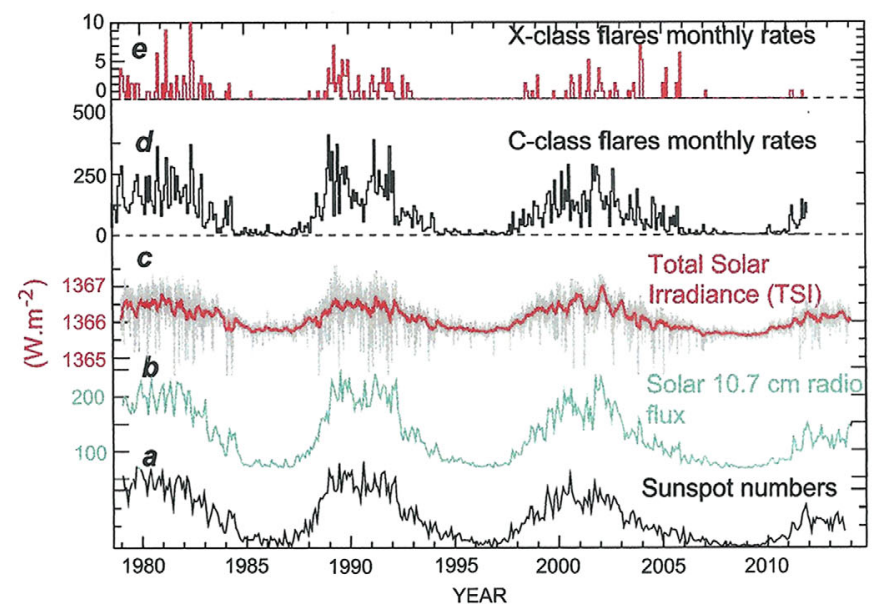

(b)

Fig. 2 (a) The various aspects of solar variability during the time of the phase transition (ref. M. Lockwood; published in de Jager 2012). (b) The time-variation of several sunspot-associated variables. Minimum values are obtained in $2008.5 \pm 0.5$. The diagram is copied from Balogh et al. (2014), where the references to the sources of the five variables are given too

as compared with earlier measurements. During the past century the current opinion was that the umbral field strength of sunspots varied only little in the course of time. This viewpoint changed during and preceding the Transition, primarily due to the use Fe I $1564.8 \mathrm{~nm}$. These 


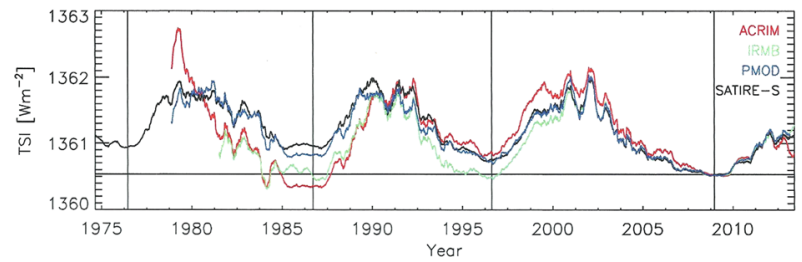

Fig. 3 The total solar irradiance (measurements from four monitors). Between cycles \#21 and \#24 the average TSI went through a minimum that may have been deeper than the average value of most of that of the foregoing ones (Yeo et al. 2014). Vertical lines mark the time of sunspot cycle minima

data were obtained by measuring the Zeeman splitting of this near-infrared Fe I line from spectra taken at the McMath-Pierce telescope. In spot umbrae this line is always completely split and it thus directly yields field strength and not flux (as given by other magnetographs). The spatial umbral resolution was 2.5 arcsec, to which seeing effects should be added.

In papers initiated by Penn and Livingston (2006, 2009), cf. also, Livingston et al. (2012), Watson et al. (2009, 2014), Livingston and Watson (2015), Rezaie et al. (2015), there was initial evidence that the average maximum umbral magnetic field strength decreased steadily from 1999-2005. This decrease is now understood to have been biased by a then observational interest in large spots. After 2005, and through to the present (2016), all spots on the visible disk were observed (in the allotted telescope time of a few days per month) and after 2010 the average approached a daily value of slightly above $2000 \mathrm{G}$, as shown in Fig. 4a. This result was confirmed in broad outline by Watson et al. (2009, 2014); cf. Fig. 4b.

Starting in 2010 the measurements of Livingston et al. were taken exclusively in the darkest position of each umbra, a few times per month, and in general one set of observations each day. The results for 4338 spots are given in Fig. 4c (which yields an average of $2070 \pm$ $20 \mathrm{G}$ ). The technical details of these observations are the same as those for Fig. 4a (but for the early large spot bias of Fig. 4a). Figure $4 c$ shows the distribution around the average constant field. Because these data are confined to the darkest parts of the umbrae, they are not comparable to those that are at the basis of Fig. 4b, where the averages over the umbrae were taken. But essentially, Fig. 4c confirmed the Penn-Livingston results of the low average umbral field strength after $\sim 2010$. Figure $4 \mathrm{e}$ is the same as Fig. $4 \mathrm{c}$ but updated to March 2016.

The initial belief was that the decrease to around $2000 \mathrm{G}$ during the Transition was a unique property of the Transition, but that conclusion was reformulated on basis of observations published by Pevtsov et al. (2012) and Nagovitsyn et al. (2012); cf. Fig. 4d. They found, from observations covering the past half-century, that the average maximum umbral field strength is virtually never constant, contrary to what had always implicitly been assumed by the solar community. An extended series of field flux measurements over the past five decades yields evidence that the cyclic diminishing effect is part of a periodic phenomenon: in successive Schwabe cycles the umbral maximum field flux goes up- and downwards, in agreement with the sunspot number. But during the Transition the decrease was more clearly evident. Figure 4d (copied from Nagovitsyn et al. 2012) should be compared with Figs. $4 \mathrm{a}, 4 \mathrm{~b}$ and $4 \mathrm{c}$. It also shows the decrease of the umbral field strength to $\sim 2000 \mathrm{G}$ between 1998 and 2010 .

We should say something about pores. Pores are traditionally defined as small dark umbrae without attached penumbrae. They are numerous and are seen in ground-based telescopes such as the McMath. But high resolution space observations indicate that tiny pores often have tiny fragments of a penumbra. The McMath observations after 2005 include 


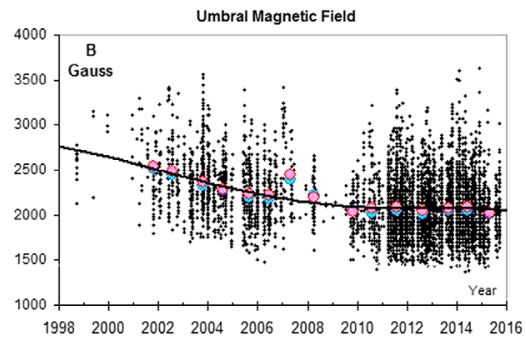

(a)

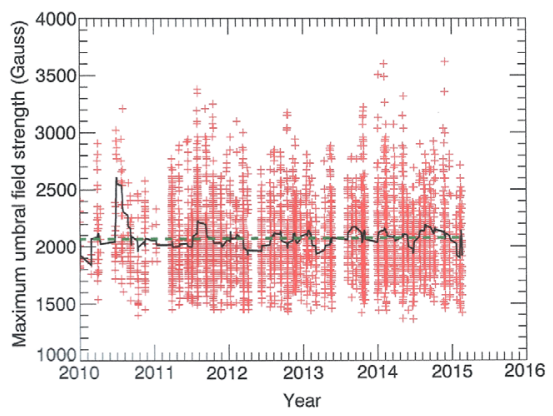

(c)

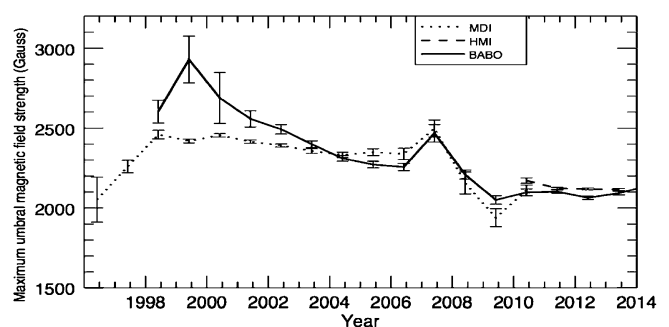

(b)

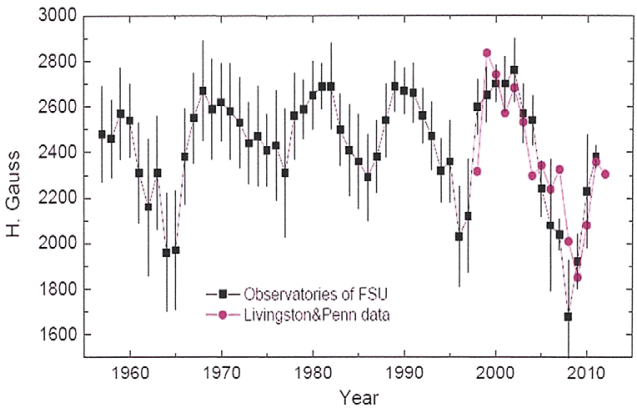

(d)

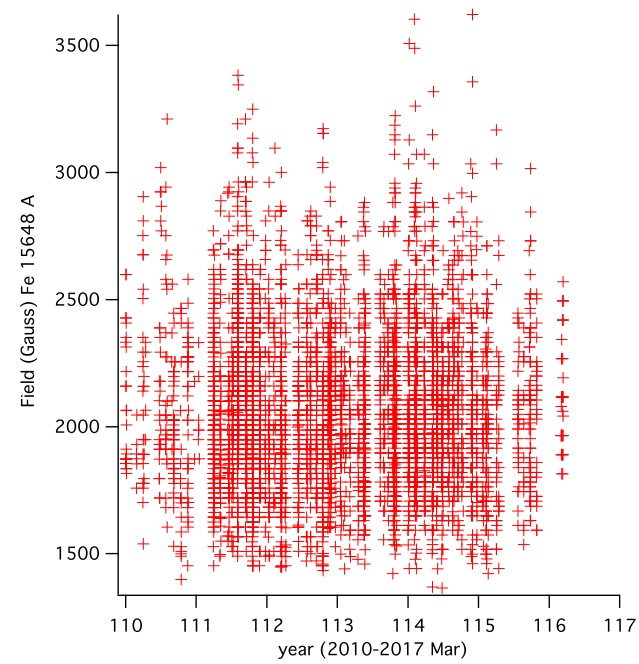

(e)

Fig. 4 (a) Kitt-Peak near-IR umbral magnetic field strength between 1999 ans 2015. After 2005 it stayed at a nearly constant value. These plots, made by Leif Svalgaard represent an improved version of a similar diagram published by Livingston et al. (2012). (b) Annual averaged magnetic fields in the darkest parts of sunspot umbrae for three different data sets (Space Obs.: MDI, HMI), and ground based (Babo $=$ Kitt Peak). From Watson et al. (2014). (c) Individual Fe $1564.8 \mathrm{~nm}$ maximum umbral magnetic field strength measured between 2010 and 2015. The average value is $2070 \pm 20 \mathrm{G}$. Plots made by Fraser Watson. Ref. W. Livingston. (d) Long-term variation. Umbral magnetic field strengths as determined by Penn and Livingston (2009), Livingston et al. (2012) and by various stations in Russia. Drawing copied from Nagovitsyn et al. (2012). (e) Same as (c) but updated to March, 2016 
"pores" whether or not we see penumbrae. Pores are treated herein the same way as sunspots. We should add that pores can be missed if the seeing is inadequate.

The data of Fig. 4a show a flat trend of about $2200 \mathrm{G}$ after 2005. A comparison of these data with those of Fig. 4d may suggest a difference between the two series of measurements but that is hard to prove because the one series of measurements virtually ends where the other starts. In the too short period of overlap between Figs. $4 \mathrm{a}$ and $4 \mathrm{~d}$ there are only small differences. That makes one wonder about the precise values in the years of overlap. It is only to be regretted that there is no better overlap between the two series of measurements.

We conclude that the image of Fig. $4 \mathrm{~d}$ is confirmed in broad outline by Figs. $4 \mathrm{a}, 4 \mathrm{c}$, and $4 \mathrm{e}$.

Before going to an interpretation of the data we need to add a few lines on their delicate character. The data of Fig. 4c are based on observations in sunspot umbrae, approximately 5 to 6 days per month. One observation is made in each spot that was visible, at the darkest position in their umbrae. Therefore, as said above, these observations are not compatible with those shown in Figs. 4a, 4b and $4 d$. Figure $4 \mathrm{c}$ is interesting by itself in that it displays the day-to-day means with their overall average (the constant dashed line).

After these remarks we conclude: Based on available information, as summarized in Figs. $4 \mathrm{~b}$ and $4 \mathrm{~d}$ we forward that, up to about 2002 there was a periodic variation with an amplitude of $\sim 300 \mathrm{G}$ around an average value of $\sim 2500 \mathrm{G}$. A general decrease down to about $2100 \mathrm{G}$ started before 2005 . That decrease ended around 2010 when the maximum umbral field strength had reached a value as low as $\sim 2000 \mathrm{G}$. (Precise measurements at selected darkest points of the umbrae yield a practically constant daily average of $2070 \mathrm{G}$ ). The maximum umbral field strength stayed virtually constant, at least till 2016. An important piece of additional of information is that the minimum of cycle 23 is not the deepest that is possible (Muñoz-Jaramillo et al. 2015). The lowest value of the umbral magnetic field as measured with the traditional Fe $1564.8 \mathrm{~nm}$ line is 1500 Gauss. This value is constant, independent of the cycle and it applies to all spots, from pores to larger ones.

The intriguing question is how this will continue in coming years.

Summarizing these conclusions: Eleven-year variations of the average umbral magnetic field values are apparently a common phenomenon but the minimum value was more pronounced and lasted longer during the recent Transition, which shows in Figs. 4 as an extended minimum. Nagovitsyn et al. (2012) give evidence that the Livingston-Penn effect is regulated by the relative contribution of small and large sunspots in the ensemble of sunspots, in such a way that the relative number of smaller spots was larger during the extended minimum. They stress that there is no monotonous centennial decline and that the umbral magnetic field measurements just exhibit cyclic variations.

This conclusion is confirmed in the various components of Fig. 4.

\section{Cosmic Ray Observations}

"The intensity of cosmic rays directly reflects the various solar cycle variations, from the well-known 11 and 22 year cycles, with the reversal of the solar magnetic field at extreme solar maximum to highly temporal variations like proton flares, Forbush decreases, corotating interaction regions and a variety of propagating diffusion barriers. .. Recently, the time-dependent extent and the dynamics of the heliosphere, in particular the role of the heliosheath and the heliopause, have been emphasized as important to very long-time space climate", as quoted from Potgieter (2008). Hence, the overall variation of the intensity of 
cosmic rays at the Earth depends on various aspects of solar, interplanetary and heliospheric activity. For details cf. the review by Potgieter (2013).

The modeling of the global heliosphere with comprehensive MHD models indicates that the heliosphere is highly asymmetric in terms of a nose-tail and even a north-south (meridional) direction and that conditions in the interstellar medium could influence the extent of the heliosphere (e.g. Borrmann and Fichtner 2005), and therefore also cosmic rays over millennia. For a comprehensive review on several aspects of space climate related to cosmic rays, cf. Scherer et al. (2006) and for a comprehensive review of solar activity over millennia, Usoskin (2013). Cosmogenic radionuclides also provide records of the paleocosmic radiation that extend to more than 103 centuries into the past and are as such indicators of changes in space climate (e.g. Beer et al. 2013). Studies of cosmic ray variations over the past 9400 years, and their implications regarding solar activity and the solar dynamo are also reported (e.g. McCracken et al. 2013).

The long-term variation as actually observed by cosmic ray detectors on ground level (called neutron monitors) is shown in Fig. 5a. This detector counts all cosmic rays with energies above about $7 \mathrm{GeV}$. The plots are shown together with sunspot numbers and the tilt angle of the wavy heliospheric current sheet (HCS). The latter is an excellent proxy for solar activity from charged particle point of view and leads to the distinct profiles which characterize the 22-year cycle seen in the upper panel of Fig 5a.

Note in Fig. 5a how on top of the larger 11-year cycle, the cosmic ray intensity typically peaks in so-called $A<0$ solar magnetic field epochs (e.g. 1960s, 1980s, etc.) but being flattish during $A>0$ epochs (1970s, 1990s, etc.). The dominant modulation process in this regard is particle drifts in addition to the conventional convection, diffusion and adiabatic energy changes. During $A>0$ polarity cycles all positively particles drift toward the Earth mainly through the polar regions of the heliosphere while during $A<0$, when they drift inward mainly through the equatorial region, they encounter the wavy HCS. Consequently, this strong relation to the changing HCS waviness (tilt angles) before and after a solar minimum shaped the cosmic ray time profiles into forming peaks. This process was repeated during the 2009 solar minimum $(A<0$ cycle) but the time profile was less sharp as depicted in Fig. 5a, indicative that the last solar minimum was indeed different from previous $A<0$ cycles.

Another consequence of drifts is charge-sign dependence in cosmic ray modulation because negative particles will drift differently towards the Earth. This is a main feature in the study of how galactic cosmic rays respond to what happens to the global magnetic field of the Sun; for details, see the review by Potgieter (2014). The full reversal of the polarity of the global solar magnetic field during periods of extreme solar activity is another important aspect of how the 22-year cycle is apparent in the heliosphere.

An important aspect for the present study is the rise of the cosmic ray flux to reach in December 2009 the highest maximum that have been observed since the beginning of the space research era. This is shown in Fig. 5b for the PAMELA observations (Adriani et al. 2013; Potgieter et al. 2014). Instead of grouping with the previously observed $A<0$ spectra (in red) the December 2009 spectrum was even higher than the $A>0$ spectra (in blue) which, according to drift modulation models, should always be higher than $A<0$ cycles at energies below $500 \mathrm{MeV}$. This was even measured at ground level as shown in the top panel of Fig. 5a, although at these higher energies the effect, described here, is diminished. As noted, the shape of the intensity-time profile was far less sharply peaked around 2009 than during previous $A<0$ polarity cycles.

This is a reflection of the exceptional transparency of the interplanetary medium and heliosphere for galactic cosmic rays at that time, because of the very low level of solar activity in 2009 and the preceding years. The date, December 2009, confirms the conclusion 


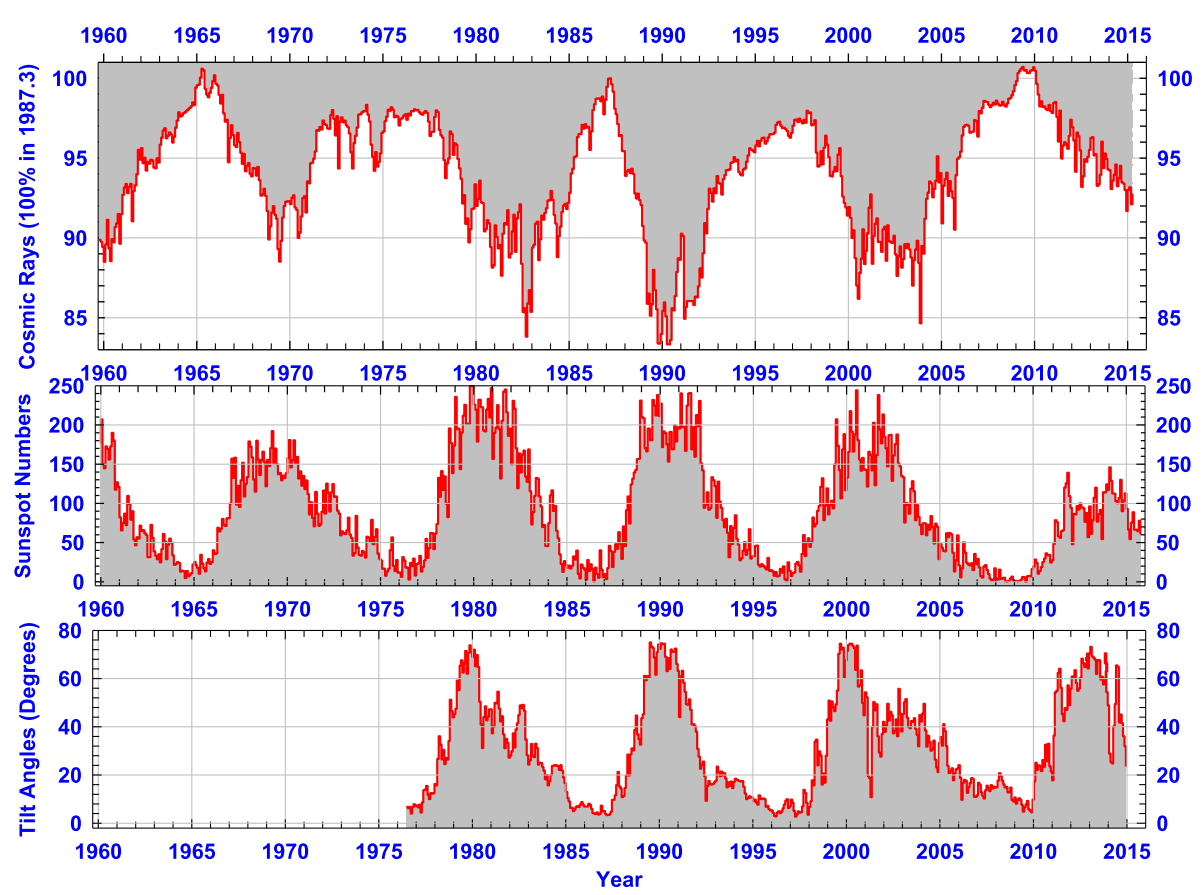

(a)

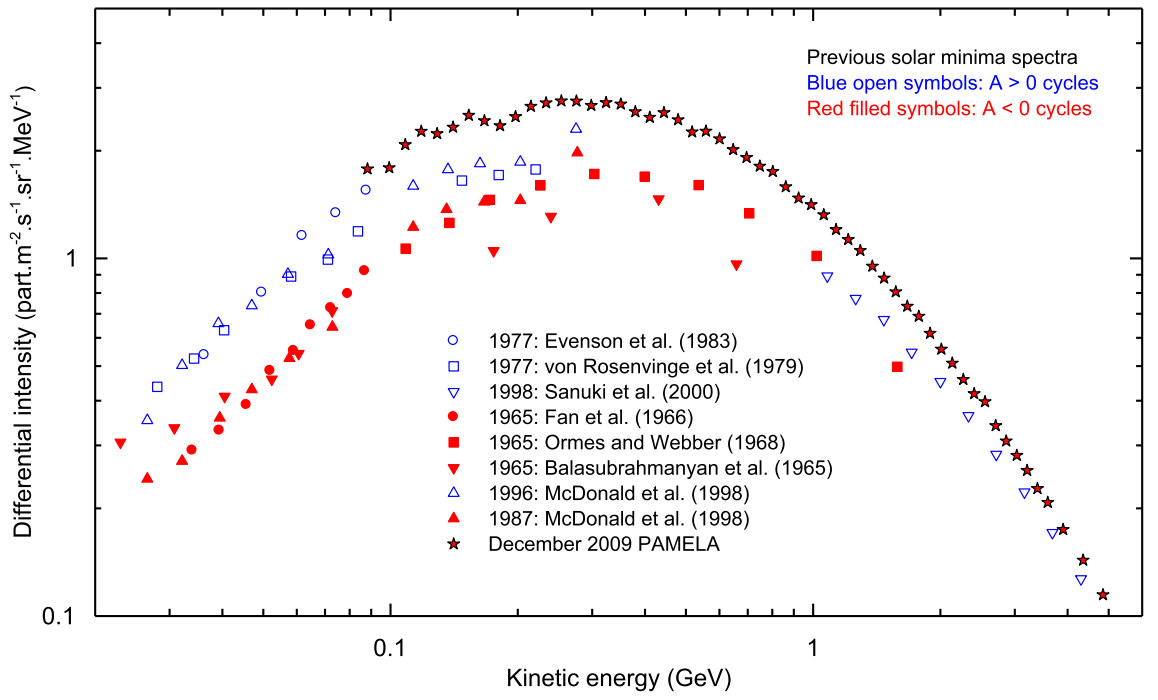

(b)

Fig. 5 (a) Top panel: Cosmic ray data observed by the neutron monitor in Hermanus, South Africa, normalized to 100 for March 1987. (See also http://www.nwu.ac.za/neutron-monitor-data.) Middle panel: Sunspot numbers since 1960; see for example http://www.sidc.be/silso/datafiles. Bottom panel: Tilt angle data as an indication of the waviness of the heliospheric current sheet; see http://wso.stanford.edu. (b) Galactic proton spectra observed over the past five decades during solar minimum activity. The $A<0$ magnetic polarity cycles are shown in red and the $A>0$ cycles in blue. The PAMELA spectrum for the end of 2009 is indicated by the star-symbols and is the highest cosmic ray spectrum observed since the beginning of the space research era. References to these observations as shown in the legend are listed by Strauss and Potgieter (2014) 
of Figs. 2a and $2 b$ that the solar particle flux reached a minimum in $2009 \pm 0.5$. In addition to PAMELA observations, Mewaldt et al. (2010) reported that cosmic rays with high rigidity reached record setting intensities during this time, while Heber et al. (2009) and McDonald et al. (2010) also noted this extraordinary event from space bound observations. It followed from these cosmic ray observations for this period that the delay between the time for minimum sunspot numbers and maximum cosmic ray intensities was at least three times longer than during previous even numbered solar cycles (see also e.g., Kane 2011; Aslam and Badruddin 2012; Mewaldt 2013).

It can also be noted from Fig. 5a that sunspot numbers (middle panel) have been very low during the present period of maximum solar activity, indicating that the Sun has indeed been quieter than during recent previous cycles, even during solar maximum periods and that cosmic rays are responding accordingly. The time-profile of the tilt angles, on the other hand, does not reveal a quieter Sun suggesting that although the Sun becomes overall quieter, with global solar wind turbulence significantly less than the average value, the solar magnetic field still follows the usual time behavior. This could be interpreted that even during an epoch such as the Maunder Minimum the polarity of the solar magnetic field had kept changing in a 22-year fashion.

However, the magnitude $B$ of the solar magnetic field observed at the Earth decreased significantly from 2006 to 2009 to become the lowest observed so far. This has contributed significantly to the observed higher cosmic rays during this period because cosmic ray diffusion and drifts scale essentially as $1 / B$. Svalgaard and Cliver (2007) proposed that $B$ at Earth has a 'floor' value of $\approx 46$ microgauss in yearly averages, which should be obtained during solar minima. They attributed the floor to a constant baseline solar open flux. In 2008 to 2009 , the notion of such a floor was undercut by annual averages of $\sim 40$ microgauss. Cliver and Ling (2011) revised both the level and the concept of such a floor and concluded that $B \sim 28$ microgauss could be a 'floor' value. They suggested that the source of the floor in $B$ is the slow solar wind; the Earth was in slow solar-wind flows $\sim 70 \%$ of the time during 2009. The decrease in the tilt angle was, in contrast, rather ordinary: it had not decreased as rapidly as the magnitude of the heliomagnetic field at Earth during this period, but eventually also reached a minimum value at the end of 2009 .

The large decreasing and increasing steps over relatively short periods shown in the upper panel of Fig. 5a is indicative of large transients originating from the Sun and that, when propagating throughout the heliosphere, subsequently disturb the quiet solar minimum conditions and eventually lead to a minimum cosmic ray flux at the Earth during periods of extreme solar activity. In this regard a prominent role is played by Coronal Mass Ejections (CME) as indicated by Cliver and Ling (2001a). These CME's are mostly correlated to the equatorial solar activity but can cause, through merging and interaction, cosmic ray regions to extend towards higher heliolatitudes and throughout the heliosphere (see e.g. Potgieter 1993). A significant decrease in the number of CMEs was observed in 2007-2009 (Vourlidas et al. 2010) which means that these cosmic rays encountered fewer barriers into the inner heliosphere so that significantly fewer transients such as Forbush decreases had been observed during the present solar cycle.

\section{Unipolar Magnetic Regions; Their Significance for Solar Variability and Their Relation to Coronal Holes}

In this Section we consider the origin and development of the solar large unipolar magnetic regions, and their relation to the coronal holes, their Hale boundaries (Svalgaard and Wilcox 
1976) and their rest latitudes (Makarov et al. 2004) during the past Grand Maximum of solar activity.

Distinction should be made between apparent and real unipolar regions. There are many seemingly unipolar regions on the sun, that are so small that their unipolar character can be negated nor confirmed. Lamb et al. (2008) find that the majority of the particle flux of the quiet sun originates from areas of coalescence of previously existing but unresolved flux, that show up as arcsecond structures. They also estimate that their rate of coalescence, integrated over the sun, is $7 \times 10^{21}$ Maxwell/hr. They further suggest that "currently unresolved emergences may be at least as important as ephemeral regions emergences to the total overall flux budget".

Next to these quasi-unipolar regions there are the larger real unipolar regions. They have field strengths of a few gauss. Their latitude bounds are fairly similar to those of the active regions and they encompass between one third and one tenth of the solar surface. Their fundamental role in governing solar variability has been grossly underestimated for a long time and was only realized quite recently.

We describe their discovery and significance. With the increased observational possibilities of the fifties of the past century, Babcock and Babcock (1955), (cf. also Simpson et al. 1955) detected large areas on the sun that were characterized by only one magnetic polarity, the unipolar magnetic field regions. Since their average field strengths are only of the order of a few Gauss, their observations demand highly developed instrumental facilities, and that was the reason why their study progressed only slowly. Initially this even led to a wrong interpretation. Leighton (1964) suggested that they originate out of bipolar active regions when these are decaying and that idea found initial support in observations by Bumba and Howard (1965). These authors found that their latitudinal distribution coincided fairly well with that of the bipolar active regions. Further support of that suggestion was presented by Giovanelli (1982).

It was at about the same time that McIntosh and Wilson (1985), also guided by earlier work from Svalgaard and Wilcox (1976) found that the above scenario cannot be correct, if only for the reason that unipolar magnetic field regions do appear at the surface before the disappearance of spot groups. Later investigations gradually showed the fundamental importance of these regions for solar variability, particularly for the origin and developments of sunspot pairs, cf. Solanki (2003), Akasofu (2014, 2015).

The present views are represented by a schematic diagram published by Akasofu (2015) that is partly based on work by McIntosh (1981). It is reproduced here as Fig. 6. Unipolar magnetic regions occur on the solar surface in an alternating positive-negative sequence. These two parts are separated by a neutral line, the Hale boundary. The spots of the two opposed polarities of sunspot groups occur on the two sides of the Hale boundary. Single spots of the same polarity as the unipolar magnetic regions may occur inside the unipolar magnetic regions. The difference between the even- and odd-numbered solar cycles (the Hale law) and also the different behavior on either side of the equator are illustrated by the two parts of Fig. 6. A further comment is that only the latitudinal dependence is shown in the diagrams. With regard to this, the examination of a large number of magnetic maps, depending on the polarity of the dipole field (sunspot cycle) shows that one of the unipolar regions is often, but not always, connected to the polar field. Because the relationship between the polar and unipolar fields is badly known (but for the polar migration of unipolar field), the diagram does not include the polar field. Also for that reason Fig. 6 is a schematic representation.

The systematic equatorward drift of the region of occurrence of sunspots (the 'butterfly diagram') may find an explanation in a study by Nakagawa (1971). In his model largescale deep-seated whirling circulation patterns, oriented along the meridians, occur on either 
Fig. 6 Schematic diagram showing the relation between positive and negative unipolar regions and sunspot pairs during even and odd sunspot cycles. Plotted are the successive Schwabe cycles in the conventional way: latitude against time. Copied from Akasofu (2015)

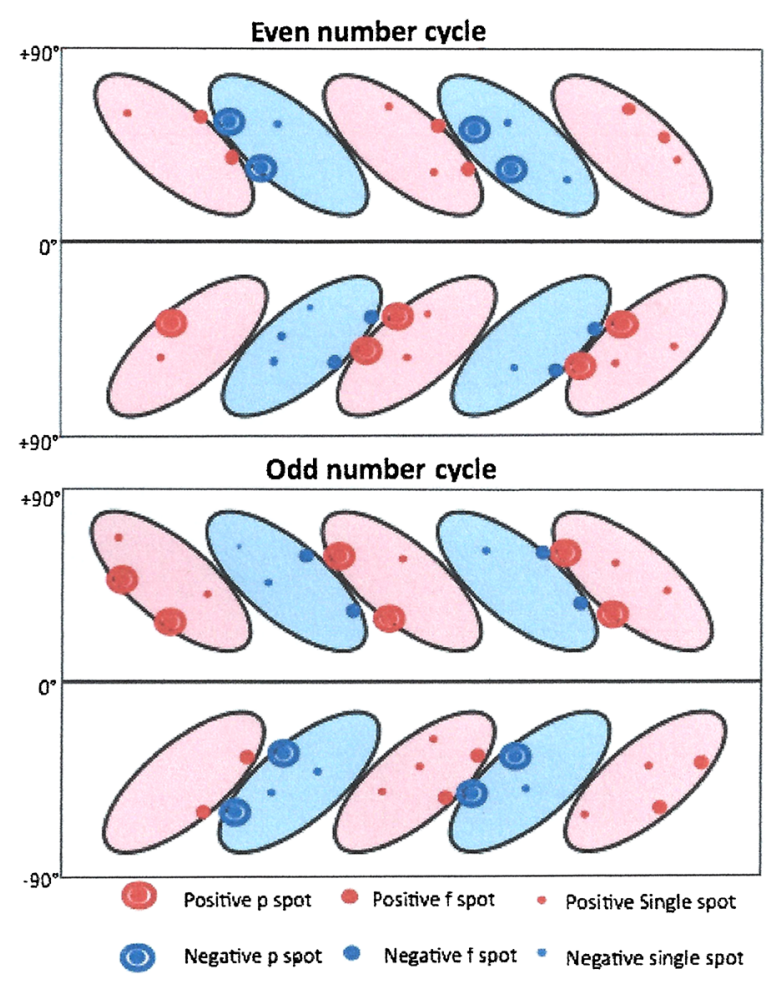

hemisphere. While the surface part of the whirl is magnetically positive, the inverse is the case with the deeper part of the whirl and inversely so. The surface part shows as magnetically neutral regions. It is these deeper whirls that are suggested to be responsible for the butterfly effect.

Makarov et al. (2004) found that new unipolar regions are generated during each cycle in a more or less symmetrical way near the equator (in the beginning: very irregularly). They have opposite signs in the two opposite hemispheres. The borderline between two large scale unipolar regions is an apparently neutral line (neutral in the line of sight).

Zwaan (1987) was one of the first to emphasize the role of unipolar magnetic regions in the formation of coronal holes. These latter structures had already been discovered by Waldmeier (1957) in HeI observations but they only came out clearly when X ray observations became available. Their magnetic fields are unobservable or weak and in the latter case they are predominantly unipolar. The field lines stretch out away from the sun, to result in diverging open field lines (Zwaan 1987). They are present during the major part of the solar cycle but are most pronounced during sunspot minimum. As a fairly general rule they are absent around maximum sunspot number. Bohlin (1977) showed that a new coronal hole is formed after the emergence of a nearby active region. Later (quotation from Zwaan 1987 ) it became apparent that "a large open magnetic structure which may show a coronal hole during part of its existence is usually associated with a succession of different active regions", and that "long-lived coronal holes are caused by a specific pattern in the emergence of active regions". Transient jets occur in polar coronal holes; some of them have a motion that is transverse to the outflow direction. In the early phase of the Transition the jet-base ephemeral active regions in the coronal holes had a preferred east-west direction, that changes into the opposite in the later part, around late 2008 (Savcheva et al. 2009). 


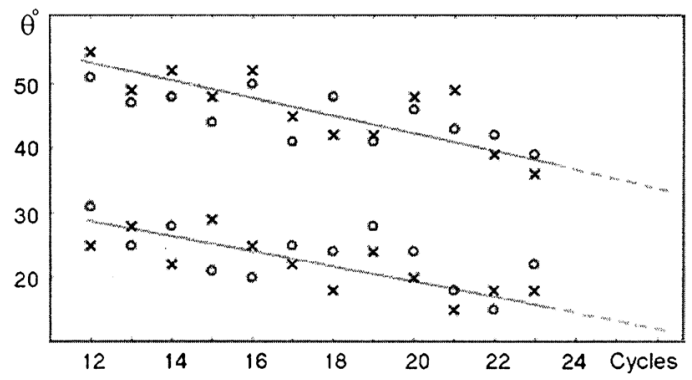

Fig. 7 The rest latitudes of the large scale unipolar regions have gradually decreased and thus moved near to the equator during the past Grand Maximum - was the new solar Episode born inside the previous one? The diagram (Makarov et al. 2004) gives the annual mean latitudes of the high- and low-latitude boundaries of the large-scale magnetic areas, where crosses refer to the northern and circles to the southern hemispheres. At the time of the Transition it was already near to the solar equator

This demonstrates that coronal holes find their source in the unipolar magnetic regions. The importance of coronal holes versus Coronal Mass Ejections as sources for cosmic ray modulation was studied by Cliver and Ling (2001b). The latter are more important than the former, but the latitude of the coronal hole plays a strong role in the sense that the open flux from high latitudes is strongest correlated with cosmic ray intensities.

A remarkable effect, most probably related to the present theme of the Transition was mentioned by Makarov et al. (2004). The position of the low-latitude termination of the Hale boundary came gradually closer to the equator in the course of the past 20th century Grand Maximum. During cycle 23, hence close to the time of the Transition, it was already nearing the equator (Fig. 7).

Since we now know that these regions are the areas of birth of coronal holes we conclude that during cycle 24 (and later?) coronal holes will extend down to very low latitudes, even to close to the equator. The coronal holes are the sources of (mainly weakly magnetic) solar winds and this generates the conclusion that the solar wind must be strong and mainly with a low magnetic flux in (the) coming cycle(s). This has indeed been observed (Lockwood et al. 2009, Lockwood 2013), and that finding is a fine confirmation of the Makarov-TlatovCallebaut observations.

This phenomenon may, perhaps only partly, be related to the latitude drift of sunspots (Richardson and Schwarzschild 1953; Tuominen 1954): at latitudes below $26^{\circ}$ the spots move equatorward, the faster when closer to the equator, with an extreme value of 0.017 degrees per day at $2^{\circ}$ latitude. At higher latitudes they move poleward, but with slower speed.

During the past few Schwabe cycles the lower rest latitudes have arrived at latitudes that are fairly close to the solar equator, leaving only little space for a large unipolar region between it and the equator. The opposite situation applies in the other hemisphere. This observation too may be relevant for understanding the recent phase transition.

\section{Decreasing Equatorial Activity Since $\sim 1970$}

In line with the foregoing is the general decrease of equatorial activity in the second half of the past century, as illustrated in Figs. 8 and 9.

The maximum field strength of the toroidal field as measured by $R_{\max }$ was steadily decreasing, at least during the past two or three solar cycles. This decrease is not only seen in 


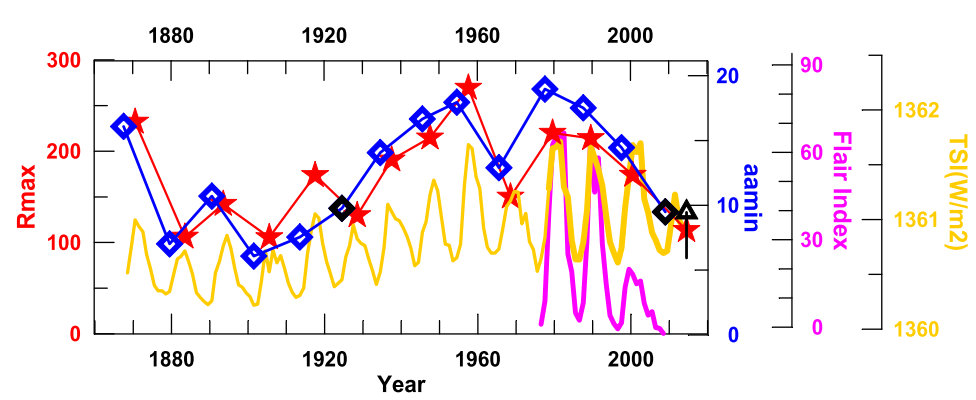

Fig. 8 Sunspot number at maxima and geomagnetic index at minima (stars and diamonds, respectively), flare index (blue curve) and total solar irradiance (orange curve), after 1978 when direct observations started (thick drawn curve) and prior to that (extrapolated; dashed curve). The red star is the prediction of sunspot maximum \#24 (de Jager and Duhau 2011). The two black diamonds are placed at dates of the first and last polar maxima of the 20th century Grand Maximum. This diagram plots the Clete et al. $(2014,2015)$ sunspot numbers against the years

$R_{\max }$ but also in those other variables of which the variability is driven by the poloidal field variations of which the strength is measured by $a a_{\mathrm{min}}$. The Total Solar Irradiance (TSI) has been nearly constant during that time and its value in 2011 was still well above the extrapolated value of the maximum of solar cycle \#12, a cycle that is quite similar to \#24. We note that the TSI has been measured only during the last three cycles, but it has been extrapolated downward on basis of its good correlation with the sunspot number. The number of flares (blue curve) decreased strongly after sunspot cycle 22 .

The progressive decline of sunspot activity prior to the Transition is not restricted to the present transition between the 20th century Grand Maximum and the subsequent Episode. There was also "a progressive decline of solar activity before the onset of the Maunder Minimum” (Clette et al. 2014).

A year before the maximum of cycle 24 the TSI was still at a level comparable with that at solar maximum of cycle 20 and well above its value at solar maximum \#12. The above differences in the variables may have relevance to the matter discussed in the preceding Section, where we described the gradual equatorward movement of the rest latitudes of the large-scale unipolar regions. Later in this paper (Sect. 11 and subsequent sections) we investigate to what degree these movements of solar field-related parameters may be interpreted as forerunners of the Transition.

\section{Polar Variability in the Framework of the Total Solar Variation}

In the later part of the polar cycle the unipolar magnetic regions gradually move poleward following and replacing the previously present unipolar large region (of opposite sign), each in its own hemisphere. Ultimately (in the subsequent cycle) the large scale unipolar regions disappear near their poles.

The poleward motion of the unipolar regions can be visualized in Fig. 9a, a diagram due to Ananthakrishnan (as early as 1954; cf. also the modern version by Hathaway in Fig. 9b). These diagrams show the distribution of prominences as a function of latitude and time. The rationale of this statement is that many prominences mark the boundaries of opposite magnetic fields (Fig. 9). This diagram also makes clear how and why the unipolar magnetic areas change into polar holes when arriving at higher latitudes. "The poleward motion of 


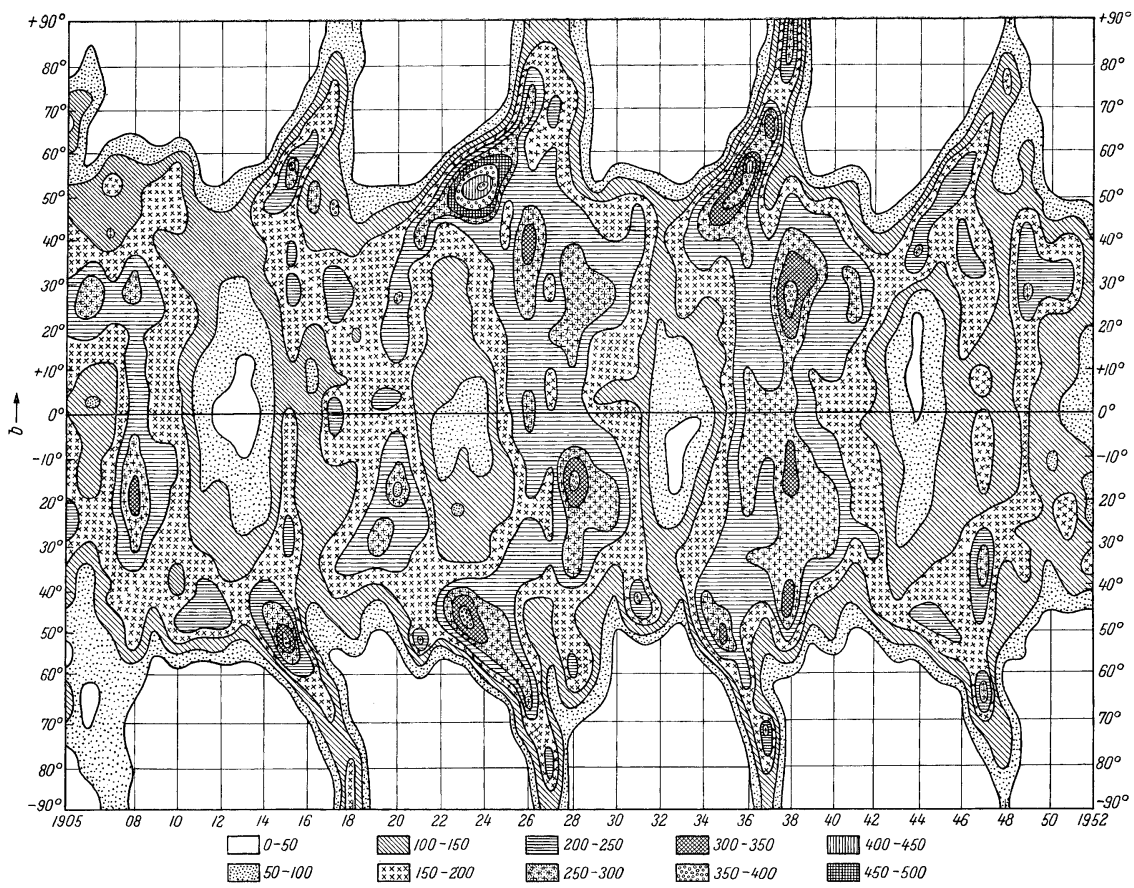

(a)

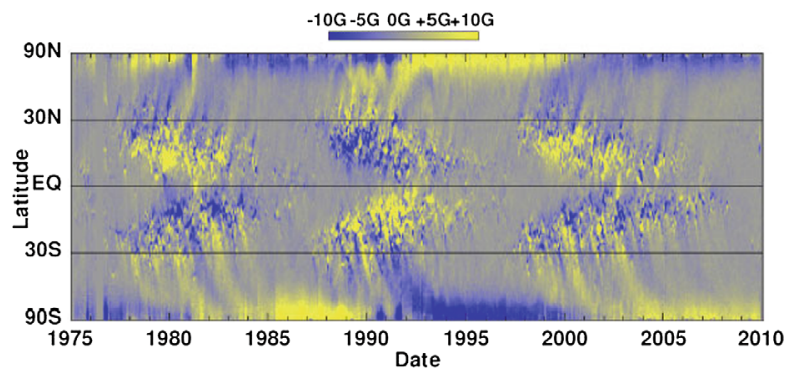

(b)

Fig. 9 (a) Isopleths of mean daily prominence area from 1905 till 1952. Remarkable are the equatorward motion of the major prominence area and the poleward motion of the high-latitude prominence zones. From Ananthakrishnan (1954). (b) A modern version of (a) that includes the recent transition; a magnetic butterfly diagram from the longitudinal averaged magnetic fields, based on observations at Kitt Peak and from SOHO. Copied from Hathaway (2010)

the solar prominence areas (and the green line emission) is connected with polarity reversal and the ensuing development of the polar fields" (Cliver 2014).

Just like the equatorial region, the polar area is a seat of various manifestations of magnetic fields. These are the polar bright points and the associated ephemeral magnetic regions and polar faculae. An important feature is the coronal holes. These are non-magnetic regions or at best regions with a small magnetic field that are mainly situated in polar areas but that do sometimes extend down to fairly low latitudes as already mentioned in the preceding Section (Zwaan 1987; Makarov et al. 2004; Lockwood et al. 2009; Lockwood 2013). 


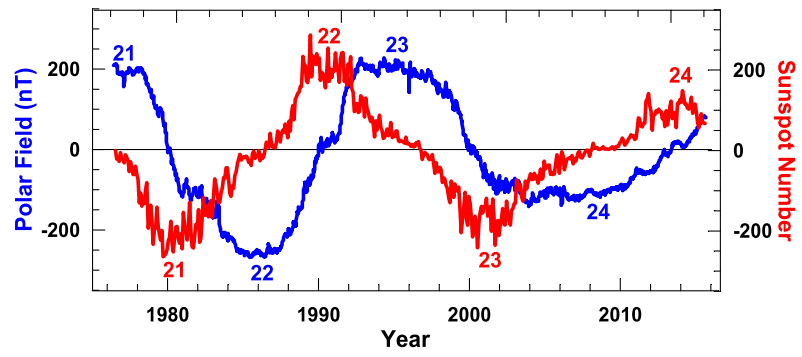

Fig. 10 The Hale magnetic cycle in the north-south average of the polar field, as observed at the Wilcox Solar Observatory (http://wso.stanford.edu) in the interval 1975.5-2010 (red; 1 Tesla = 10,000 Gauss) and the monthly values of MSIS sunspot number curve (blue) to which the sign is assigned according to the numbering of successive Schwabe cycles. The polar maxima are numbered by the standard numbers of the next following sunspot maxima. (This is a copy of Fig. 1 in de Jager and Duhau 2012)

The coronal holes are the seats of solar winds and as such they can affect terrestrial climate by their particle flow. Lockwood et al. (2009) have shown that around 2009 the interplanetary magnetic field strength obtained its smallest value since about the beginning of the 20th century. This is also shown in our Fig. 2a (open solar flux); this observation suggests the presence of extended coronal holes during the period of the Transition.

The behavior of the polar and equatorial activity during and prior to the phase transition is shown in Fig. 10. The polar magnetic flux $D M$ has been measured directly at the Wilcox Solar Observatory while the equatorial flux is given by its proxy, the sunspot number $R$. To mark the difference between successive Schwabe cycles in the framework of their Hale cycles we have allocated alternating positive and negative signs to the data from consecutive cycles, in accordance with the polarity of the polar field. From Figs. 9 and 10 we therefore conclude that the polar field strength at solar sunspot minimum is a precursor of the subsequent sunspot maximum; cf. also Schatten et al. (1978) and Svalgaard et al. (2005).

Remarkable are the differences between the present Schwabe cycle (\#24) and the two preceding ones in the period between $\sim 2000$ and 2010. The maximum of sunspot cycle \#23 is lower than that of the previous ones and the value of $R$ flattens down to close to zero after about 2007. At the same time the polar cycle after $\sim 2002$ is quite lower than the previous ones and seems to stay at about a constant value after $\sim 2004$.

Noteworthy is also the gradual decline of the total solar magnetic field over the past few decades. Its values during cycle 23 were discussed by Hoeksema (2010); a related study of the flux emergence is by Schmieder et al. (2014). Field variation during the past three cycles is summarized by Lee et al. (2011) from whom we copied our Fig. 11. That diagram shows that the start of the gradual decline of the total photospheric magnetic flux is already observable around 1980. From Lee et al. (2011) we quote: The solar field is reduced over the entire surface of the sun, while the polar fields are about half those observed during the previous minimum period. Duhau (2003) remarked that solar activity was in a declining episode, at least as early as since 1993.

Another aspect of Fig. 10 is the following: The usually accepted scenario is that polar and equatorial maxima and minima alternate: maximum of the one coincides more or less, but very roughly, with the other's minimum and inversely so. Figure 10 shows that such is the case for cycles 21 and 22: there polar maxima and equatorial minima occur simultaneously in 1976 and 1986, but that is not the case for cycle 23. For that cycle polar maximum and equatorial minimum occur in 1994 and 1997 respectively. For cycle 24 the difference in time is still larger: they occur in 2004 and $\sim 2010$ respectively. 


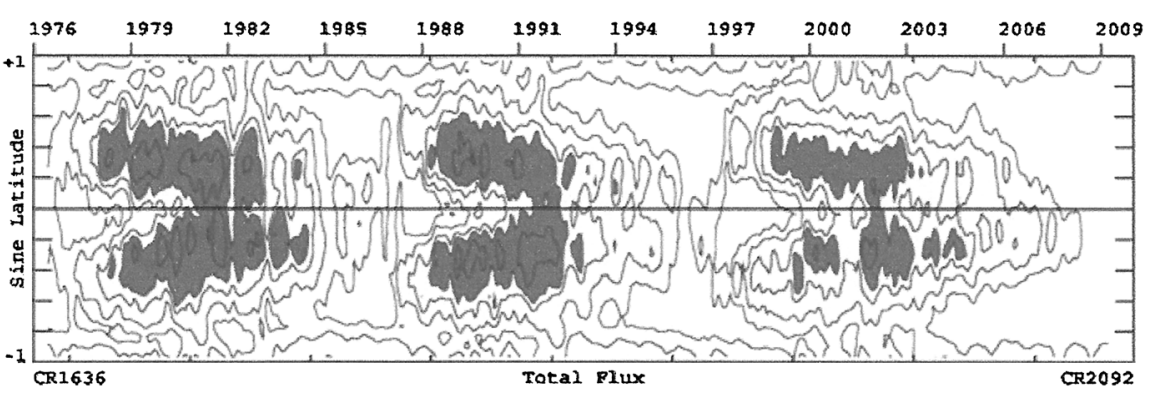

Fig. 11 Observed total photospheric magnetic field, prior to and during the Transition (longitudinal averaged). Contour levels are for values of $0.5,1,2,3,5$ and 10 Gauss. The strongest contours are shaded in dark and grey and the two weaker contours in white. Copied from Lee et al. (2011). Courtesy Stanford University, Wilcox Solar Observatory

We think that this difference may be helpful for understanding the functioning of the solar dynamo during the Transition.

Summarizing this section: We illustrate the gradual decline of the total solar magnetic field over the past few decades. At the same time the large scale unipolar regions disappeared near the poles. Figure 10 shows that the time difference between polar maximum and equatorial minimum increased in the course of cycles 22 to 24 . The first signs of the gradual decline of the photospheric magnetic flux are already recognizable as early as around 1980.

\section{Summarizing Observational Data on the Transition}

The various observations, summarized in the first part of this paper show the remarkable behavior of the sun during the period of the Transition. We defined it as the period from $\sim 2005$ through $\sim 2010$, but there are other observations indicating an earlier gradual move towards the Transition. It is even possible to state that the first indications of a precursor towards the Transition were already discernible right after the maximum of the Grand Maximum of the past century, i.e. from about 1960 onward (cf. Figs. 5, 7 and 8). This shows that the Transition is not just a short-period and impulsive feature but that it is being prepared during a longer period.

These considerations force us to investigate the various periodicities in the solar dynamo, and how they manifest themselves in the photospheric and near-photospheric aspects of solar activity. Ultimately these observations ask for an examination of the stability of the tachocline and the way it directs the functioning of the solar dynamo.

We summarize the main features of the Transition:

- During the Transition the extreme values of the equatorial and the polar magnetic fluxes occurred in 2008-2009 and 2009-2010 respectively, hence not simultaneously (Figs. 2a and $2 b$ ).

- The average umbral magnetic field value of sunspots varies cyclic in concordance with the sunspot cycle and during the Transition it took its smallest value since $\sim 1960$. It is ascribed to a larger number of weaker spots (Fig. 4a).

- Rest latitudes of unipolar magnetic fields - these being the places of birth of coronal holes - moved gradually equatorward during the past Grand Maximum and arrived close to the equator during or after cycle 24 (Fig. 7). 
- The start of the gradual decline of the photospheric magnetic field was already observable around 1980 (Fig. 11).

- Maximum sunspot numbers of successive cycles steadily decrease after the maximum (1960) of the 20th century Grand Maximum (Fig. 8).

- As a general rule the high-latitude prominence areas move poleward after the equatorward motion of the lower latitude prominences (Fig. 9).

- The polar magnetic maximum occurred during the Transition some 6 years after equatorial magnetic minimum (Fig. 10).

\section{Starting Time of the Transition}

Livingston et al. (2012) found that during the period of their observations, (from 1998 onward) the sunspot magnetic fields had up to 2002 a normal distribution, with a mean of $2436 \pm 26$ G. It was only after that epoch that the magnetic field distribution decreased, by $46 \pm 6 \mathrm{G}$ per year. That might be an indication that the Transition started as early as around the year 2002. This conclusion is supported by a diagram (not reproduced here) that shows the 'sunspot-formation fraction', as introduced by Livingston et al. (2012): a horizontal line at level unity till about 1990 that started to decrease around 1995 towards the level 0.6 in 2010. In Sect. 8 we showed that the first signs of the gradual decline of the photospheric magnetic flux are already recognizable as early as around 1980 .

Other information on the starting time of the Transition can be derived from observations of the solar microwave radiation, of which the $10.7 \mathrm{~cm}$ observations form a continuous sequence since around 1955. The variations in this flux are due to those in the equatorial facular fields, just as the Total Solar Irradiance (TSI) is. Hence, the $10.7 \mathrm{~cm}$ radiation is fully comparable to the TSI (cf. also our Fig. 3), but the amplitude of the variations in $10.7 \mathrm{~cm}$ is much more pronounced. The variations in this radiation match excellently with the frequency shift of solar internal oscillations derived from helioseismic observations (Broomhall et al. 2009), but Basu et al. (2012; cf. also Balogh et al. 2014, p. 11 and Fig. 5) found deviations from that match that were most significant for the observations at lowest frequencies $(1860-2400 \mu \mathrm{Hz})$. At these frequencies deviations are first discernible in 1995 and only later at higher frequencies.

From these data we conclude that (a) first signs of a forthcoming change of the kind of Episode were visible in the eighties of the last century, that (b) very first precursors to the Transition were already visible around 1995 and that (c) the full start came some ten years later. The implications for the driving mechanism will be discussed in the following Sections.

\section{The Phase Diagram and the Predictability of Long-Term Solar Variability}

Phase transitions occur between Grand Episodes of solar variability. A very well-known Grand Episode is the Maunder Minimum that lasted from about 1610 till about 1740. Another episode, one of the highest of the past ten thousand years (Usoskin and Mursula 2003; Solanki et al. 2004) is the recent 20th Century (Grand) Maximum, this being the period of large solar activity during the main part of the 20th century. It was characterized by strong Schwabe cycles with their associated phenomena such as an abundant number of solar flares 
and also by related features such as the increased interplanetary magnetic flux (Solanki et al. 2000). The polar flux too reached at that time higher values than ever measured before.

The question is relevant if there is anything that is systematic or predictable in the phase transitions between successive Episodes. To that end Duhau (2003) introduced a phase diagram, consisting of a plot of the proxy of the equatorial magnetic field $R_{\max }$ against that of the polar field $a a_{\min }$ (for the definition of the latter cf. Fig. 1 and the corresponding text). The resulting phase diagram is shown in the two components of Fig. 12. Here, some comments are in order.

Empirically, Duhau found that there exist a unique point in that diagram, with coordinates $R_{\max }=93.4$ (defined in the earlier system of sunspot counting) and $a a_{\min }=10.3$, that she baptized the Transition Point.

Actually, the two coordinates of this point were initially found as the average values of $R_{\max }$ resp. $a a_{\min }$ over the past few centuries. After having derived these two values (Duhau 2003), it was a fascinating addition to dynamo theory to find that in the past four centuries new solar activity episodes tend to start when the $\left(R_{\max }, a a_{\min }\right)$ curve in the phase diagram crossed this Transition Point or passed near to it (Duhau and de Jager 2010). This empirical finding of the special character of the Transition Point may have implications for our understanding of some of the mechanisms active in the solar dynamo.

Similar phase transitions took place around 1628 (onset of the Maunder Minimum), in 1740 (end of Maunder Minimum, and transition to a period of Regular Oscillations; our Table 1 gives 1719, on basis of the phase diagram of Fig. 12) in 1923/24 (onset of 20th century Grand Maximum), and the most recent one started around 2008. Other possible transitions may have occurred in 1230, 1349 and 1405 (Duhau and de Jager 2008), but these are unverifiable, being based on extrapolated, hence uncertain values of the proxy data of the magnetic fluxes: First, the $a a_{\text {min }}$ data are only known after 1844 and earlier $a a_{\text {min }}$ data have been derived by Nagovitsyn (2005) by extrapolation. But this extrapolation rests for an important part on the high degree of correlation between $R_{\max }$ and $a a_{\min }$, while the essential aspects of the phase diagram are based on the subtle differences between both. Therefore only the late (1800-present) diagram is reliable. We publish the earlier one too, for illustrative purposes.

Another comment is the following: the $R_{\max }$ data in the phase diagrams that have been published so far were always derived by using the 'classical' Zürich international sunspot numbers (ISN). We have decided to derive the present phase diagrams on basis of the new Group Numbers (GSN; Svalgaard and Schatten 2016; cf. end paragraph of Sect. 1 for the definitions). In order to eliminate relatively shortlived variations such as those due to volcanoes, el Nino, the Schwabe cycle and the like, the Svalgaard-Schatten GSN data have been smoothed with a Lowess smoothing technique, by using an 18 years smoothing interval, which is the optimal value (de Jager and Usoskin 2006). By this smoothing these fluctuations are not eliminated but they are no longer dominating.

Thus, the data figuring in the phase diagrams of Fig. 12 were derived. The Modified International Sunspot Numbers have values of the order of many tens to above one hundred while the classical International Sunspot Numbers and the associated Group Sunspot Number values are about ten times smaller. Therefore, the $R_{\max }$ value of the Transition Point, from here onward applied to the GSN data, is in the new diagrams roughly some ten times smaller than the value that was used previously when it was based on the ISN data.

The diagrams shows close passages to the transition point to occur in 1628 (onset of the Maunder Minimum) and again in 1719 (onset of the period of Regular Oscillations). Hence, according to this diagram, the Maunder Minimum terminated around 1719, although direct observations rather favor the time around 1740. And indeed, the next following nearest year 


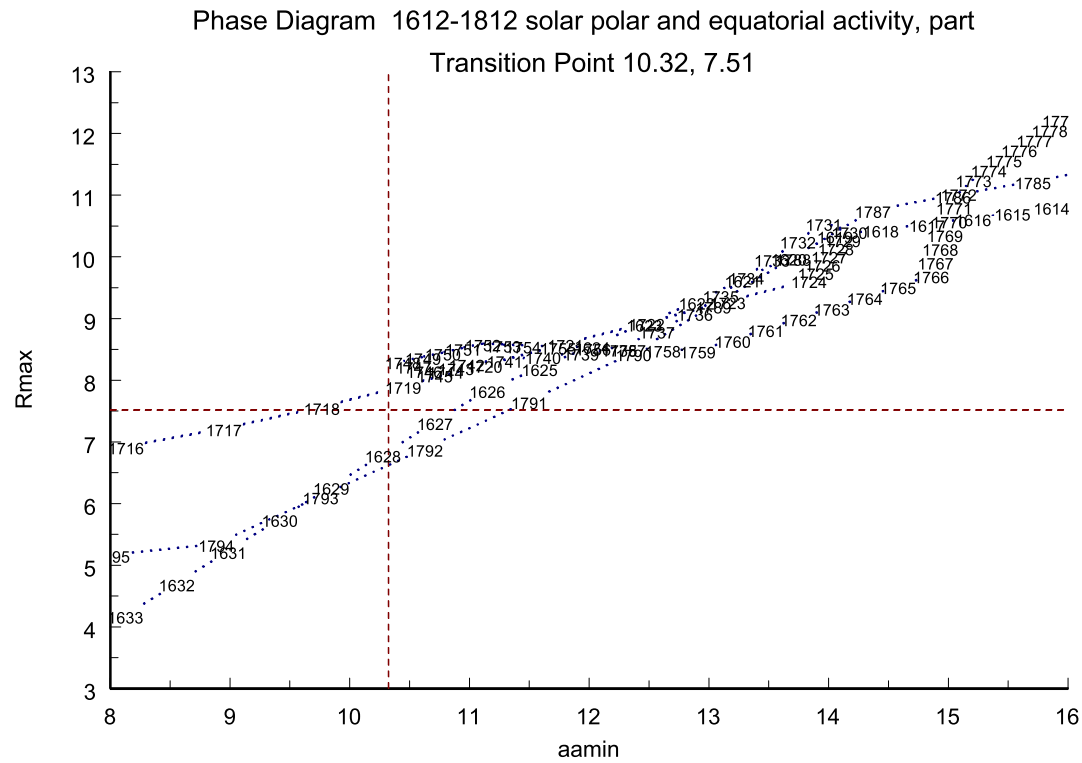

(a)

Phase Diagram 1812-2007 solar polar and equatorial activity

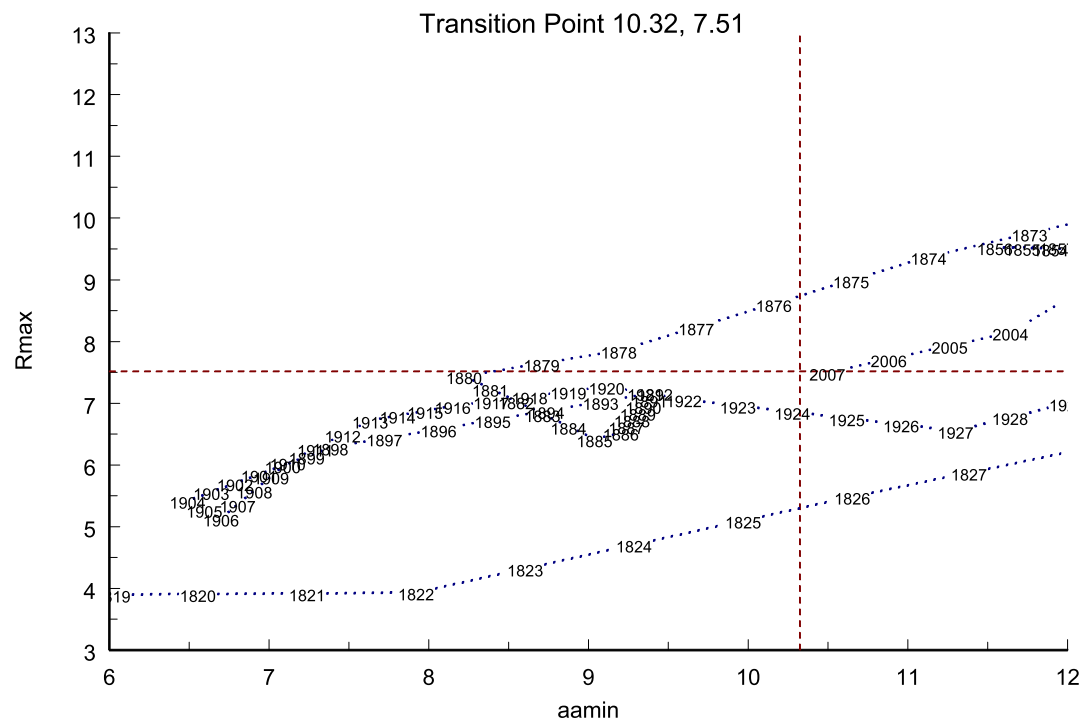

(b)

Fig. 12 Two phase diagrams, for the periods 1612-1812 and 1812-2007. These are modernized versions of diagrams published by de Jager and Nieuwenhuijzen (2013). In the present copy use is made of Lockwood's homogenized $a a_{\min }$ data (Lockwood, priv. com.), smoothed by an 18 years Lowess technique and the newly introduced Sunspot Group Numbers (Svalgaard and Schatten 2016), smoothed likewise 
Table 1 Years of closest passage to transition point and corresponding distance as defined in Eq. (1)

\begin{tabular}{lll}
\hline Year of passage & Distance & Start of \\
\hline 1627 & 0.04 & Maunder minimum \\
1628 & 0.10 & ibid \\
1719 & 0.05 & Regular oscillations \\
1924 & 0.08 & 20th century maximum \\
2007 & 0.02 & Regular oscillations (?) \\
\hline
\end{tabular}

is 1746 , but its distance to the Transition Point has then grown to about 1.5 times that of 1719. This deviating result may be due to the fact that the $a a_{\min }$ data from before 1844 are not based on direct measurements but on extrapolations (as stated above) and may, hence, not be fully reliable.

A later close passage occurred in 1924. That year does not deviate much from the one given by Lockwood et al. (2009) who found 1920 in an investigation of the open solar flux (on basis of the assumption that a Grand Maximum takes place when the 25-year average of the heliospheric modulation potential exceeds $600 \mathrm{MV}$ ). It corresponds to the onset of the Grand Maximum of the 20th century. The last passage took place in 2007-2008. It is the theme of this paper. The observations described in this paper support the evidence of the correctness of the interpretation of the phase diagram.

We next examine if there is some system in the 'close passages' to the Transition Point. To that end we define the 'distance of closest passage' (de Jager and Nieuwenhuijzen 2013) as the root of the quadratic sum of the differential $a a_{\min }$ and $R_{\max }$ values, both with respect to the coordinates of the Transition Point and both normalized by dividing them by the values of these coordinates. These new coordinates are based on the Group Numbers; they are 7.51 for $R_{\max }$ while we confirm the value 10.32 for $a a_{\min }$, which is equal to the values used in earlier studies. We calculate the distance to the Transition Point as

$$
\text { distance }=\left\{\left(\Delta R_{\max } / 7.51\right)^{2}+\left(\Delta a a_{\min } / 10.32\right)^{2}\right\}^{1 / 2} .
$$

The resulting data are specified in Table 1 .

Table 1 gives the year 1924 as the starting date for the 20th century Grand Maximum. That year is derived from the phase diagram (Fig. 12) in the way described above. We mentioned already that Lockwood et al. (2009) arrived at a similar starting year (1920) on the basis of their study of the open solar flux.

The present authors were hoping that the value of the 'distance' might, in some way be related to the character of the next grand episode but there are only weak such indications: the start of the Maunder Minimum, being the most extreme of the recent Grand Episodes, is related to the shortest distance. Clearly, a few more centuries have to pass before reliable conclusions can be taken. An essential result is that the extended transition phase, that lasted from $\sim 2005$ till $\sim 2010$, is related to the start of another Grand Episode. Hence, it marks the end of the 20th century Grand Maximum (Duhau and de Jager 2008, 2010; cf. also the review de Jager and Duhau 2012).

The next question is that of the character of the next Grand Episode. A Grand Minimum does not seem probable. Steinhilber et al. $(2009,2010)$ have shown that such type minima occur only during negative phases of the Hallstatt period of solar activity and that statement was confirmed by de Jager and Duhau (2012). The Hallstatt cycle (Clilverd et al. 2003, 2006; Muscheler et al. 2004) has a length of 2300 years, and it is, as observations show (cf. Fig. 10 of Versteegh 2005), based on the regular accumulation of Grand Minima. These accumulations repeat themselves with a periodicity of $\sim 2300$ years. Since the Hallstatt 


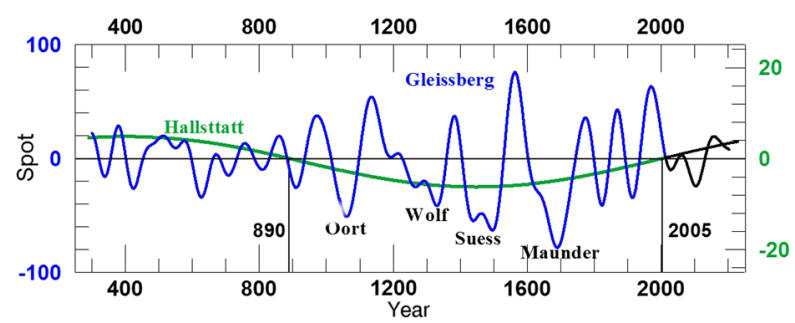

Fig. 13 The recent Hallstatt oscillation (green; right ordinate), and the Gleissberg cycle (blue; left ordinate) and their respective predictions till the year 2200 (black lines). The zero line was crossed at the beginning of the of the 21th century. These results are based on the Modified International Sunspot Numbers (MISN; Clette et al. 2014, 2015), extended backward from 1705 till 290 by the Schove (1955) time series

cycle passed through its zero line early in the 21th century (our Fig. 13) one may not expect a Grand Minimum to come. A Grand Maximum is not very probable either, on statistical grounds, for the simple reason that a succession of two such maxima has not been observed before. Therefore, another period of Regular Oscillations, such as occurred between 1740 and 1923 seems the most probable one (cf. also Duhau and de Jager 2010; de Jager and Duhau 2012). This conclusion is confirmed in a study presently underway. We interpret the recent phase transition as the crucial step towards that Episode.

Sunspot cycle 23 occurred close to the beginning of the new Episode. The next following cycle, 24, with the observed smoothed maximum value of 68 sunspot numbers - the smallest of the past century - was reached after a small but significant Gnevyshev gap (for its definition cf. Basilevskaya et al. 2006). From the more successful early forecasts of that maximum we mention e.g. Duhau (2003) who came already at an early time to an expected sunspot maximum of $87.5 \pm 23.5$ Another forecast based on similar points of departure is that of Choudhuri et al. (2007) and that of Jiang et al. (2007) who expected for the maximum sunspot number of cycle 24 values that were $30 \%$ and $35 \%$ respectively smaller than that of cycle 23, of which the maximum sunspot number was 121 . These forecasts too were only slightly higher that the observed value. Later forecasts too came closer to the observed values, e.g. Solanki and Krivova (2011) foresaw "between 60 and 100".

\section{The Tachocline; The Origin of Sunspots}

A dynamo is a physical system that transforms kinetic into magnetic energy. In this Section we restrict our discussion of the solar dynamo to the aspect that is important for the origin of sunspots. Essentially, we refer to a few properties of the Babcock-Leighton phenomenological model. In choosing that model we base ourselves also on Muñoz-Jaramillo et al. (2013a, 2013b), Cameron and Schüssler (2015) and Petrie (2015) who find that this model well covers the observational data.

The solar dynamo is situated in the outer convection zone of the sun. That zone has a depth of $\sim 2 \times 10^{5} \mathrm{~km}$ (Christensen-Dalsgaard 1991; Basu 1997; Brun and Toomre 2002; Cally 2003). In order to allow for the observed time scale of solar activity, which is of the order of 10 years, a skin depth of the relevant oscillator is needed of the order of $10^{5} \mathrm{~km}$. This is one of the reasons for identifying the dynamo with parts of the outer convection zone (review by Rüdiger 2008). At the bottom of that zone the correlation time of the turbulent cells is about equal to the value of the solar rotation period, and that is one of the reasons for situating the essential part of the dynamo in that region. Part of the dynamo is the tachocline 
which consists of the shearing layers at and below the base of the outer convection zone. The tachocline is a relatively thin, but as many observations show, not perfectly spherical layer (Elliot and Gough 1999; Antia and Basu 2011). It is 27,000 km thick at the equator and $30,000 \mathrm{~km}$ at latitude $60^{\circ}$ (Charbonneau et al. 1999). The outer boundary is prolate while the inner boundary is nearly spherical (Antia and Basu 2011). It is situated at an average depth of $215,000 \mathrm{~km}$ below the solar surface, just below the base of the outer solar convection zone, where the combination of convective motions and differential rotation powers the magnetic fields. Detached elements of the internal fields originate by kink-instability and may thereupon ascend to appear at the surface as the various elements of solar variability. These are, among other phenomena, the sunspot belt, the polar magnetic areas and the large unipolar areas.

At the tachocline level the interplay of convective motions and differential solar rotation leads to the origin of a complicated nonlinear system of magnetic fields, based on associated electric currents (Charbonneau et al. 1999; Basu and Schou 2000; Käpylä et al. 2006). The tachocline, hence, is the result of an interplay between the motions generated by the large-scale differential solar rotation and the small-scale stochastic motions due to the anisotropic turbulence generation by the convective motions in the outer solar convection zone. This interplay between motions at very different scales greatly complicates the theory of the dynamo (Brandenburg et al. 2012), which is (Weiss et al. 1984; Weiss 1988) an example of deterministic chaos. In the traditional theoretical approach the high conductivity hampers the development of large-scale flow and associated large-scale fields, but in a different approach by Tobias and Cattaneo (2013) high-resolution dynamo simulations succeed in producing large-scale structures and flows at high magnetic Reynolds numbers. In their solution propagating dynamo waves are a dominant feature of the solar cycle.

Due to the internal solar shearing motions the magnetic fields are stretched into a direction parallel to the solar equator. Thus, ultimately the system of magnetic fields becomes mainly toroidal. By continued stretching the toroidal field strength increases in the course of subsequent solar rotations. When these field strengths reach a critical value, the resulting toroidal field becomes unstable. Smaller closed loops detach from it, due to kink instability. They have smaller specific weights and hence stronger buoyancy than the surrounding solar gas, because of their high magnetic field strengths. Therefore these loops rise, to reach the solar photosphere after one or a few months. There they appear as a pair of sunspots (Solanki et al. 2004). This hypothetical scenario for the origin of sunspots is supported by helioseismic observation of sunspots on their way upward; at the time of the observations they were still as deep as 65,000 km below the solar surface (Ilonides et al. 2011).

For a quantitative analysis of the evolution of the solar magnetic field it is important to know the above mentioned critical value for which sunspot forming loops originate by kink instability of the tachocline. Early determinations are 100 kilogauss (D'Silva and Choudhuri 1993; Caligari et al. 1995 and Ruzmaikin 1998) and 45 kilogauss (Dikpati 2005). On basis of these data de Jager (2005) suggested a critical value of $80 \mathrm{kG}$. More recent work show that a minimum field $>40 \mathrm{kG}$ is needed to form solar active regions (Weber et al. 2013). Quoting from Weber: At $100 \mathrm{kG}$, the development of Omega-shaped rising loops is mainly controlled by the growth of the magnetic buoyancy instability. However, at low field strengths of $15 \mathrm{kG}$, the development of rising Omega-shaped loops is largely controlled by convective flows, and properties of the emerging loops are significantly changed compared to previous results in the absence of convection. With convection, rise times are drastically reduced (from years to a few months), loops are able to emerge at low latitudes, and tilt angles of emerging loops are consistent with Joy's Law for initial field strengths of greater than or equal to $40 \mathrm{kG}$. In a 
more recent study in which the effects of radiative heating in turbulent solar-like convection are included, Weber and Fan (2015), found that a minimum value is needed that should in any case be stronger than $60 \mathrm{kG}$. On basis of the above data we suggest to use in our further calculations a critical value of $60 \mathrm{kG}$. The uncertainty of this value is obvious; let us assume a value of $\pm 20 \mathrm{kG}$.

Extended descriptions and analyses of the dynamo are found in many review papers. We refer to the papers by Küker et al. (2001), Käpylä et al. (2006) and the reviews by Fisher et al. (2000); Tobias (2002); Ossendrijver (2003); Charbonneau (2010); de Jager and Duhau (2011); Schmieder et al. (2014); Fletcher et al. (2015). Many more papers could be mentioned but we do not consider that necessary because a treatment of dynamo theories is outside the scope and purposes of the present paper. Only one essential aspect of the dynamo theories is considered in this paper. It is the matter of rising loops that appear as sunspots and activity centers after having detached from the tachocline by kink-instability, viz. the abovedescribed mechanism that is, along with other authors, dealt with by D'Silva and Choudhuri (1993), Caligari et al. (1995), Ruzmaikin (1998), Dikpati (2005), Weber et al. (2013) and Weber and Fan (2015).

A related additional aspect is that the dynamo is a nonlinear system governed by deterministic chaos (Weiss 1988, 2010; Mininni and Gomez 2002; Charbonneau et al. 2004; Weiss and Thompson 2009). But this should be specified: Quoting Weiss (2010): While modulation leading to grand maxima and grand minima does most probably correspond to the non-linear pattern revealed in simplistic dynamo models, the small-scale turbulent motion cannot be modeled in detail. Based on an earlier study by Moss et al. (2008), Usoskin et al. (2008) could show that the occurrence of Grand Minima could be imitated in numerical experiments on the basis of a simple dynamo model with random fluctuations of the dynamo governing parameters. They also found that during the Grand Minimum (reference: the Maunder Minimum) the Schwabe cycle continues to exist, though at a very reduced scale. These studies lead us to conclude that the solar dynamo is a non-linear rather than a chaotic system.

The essential questions are here: what values of the dynamo parameters are critical in driving the grand minima? Usoskin et al. (2008) found that grand minima occur for specific values of the parameters in the dynamo equations leading to random fluctuations in the dynamo governing equations. And why does another Grand Episode start when the proxies for the polar and the equatorial magnetic fluxes arrive simultaneously at their average values (= the Transition Point)? This mere fact forces us to consider the fundamental question: which dynamo parameters were critical in driving the solar dynamo during or around the phase Transition?

\section{The Tachocline and the Transition; An Attempt to Synthesize}

We want to use the observations described earlier in this paper (cf. Sects 9 and 10), together with the considerations described in Sect. 12, to improve our understanding of the structure and functioning of the solar equatorial activity during and preceding the Transition.

Decrease of Umbral Magnetic Field Strengths Sunspots originate in kink-unstable loops that detach from the toroidal field in the tachocline. These loops thereupon rise to appear at the solar surface as sunspots after a couple of months. They can only detach from the toroidal field in the tachocline when the toroidal field in the tachocline has reached a minimum magnetic field strength. An average value of that value, derived from data given 
by various authors (summarized in the preceding section) is 60 kilogauss. That value does not depend markedly on the position of the tachocline in the sun. The umbral field strength at the time they arrive at the surface depends on the difference in gas density between the tachocline and the solar surface regions. The components of Fig. 4 shows that the average umbral field strength decreased during the 10 years of the Transition from $\sim 2700$ to $\sim 2000$ Gauss. Since the surface magnetic field in the sunspots depends on the distance traversed from the tachocline to the surface, this must imply that this distance has increased during the years of the Transition. In other words: the equatorial diameter of the tachocline has gradually decreased between the years 2000 and 2010. There are as yet (2016) no indications that it started to increase, as happened before during earlier Schwabe cycles. The emerging picture is that a least during the past Grand Maximum the average umbral field strength was oscillating, and keeping pace with the Schwabe cycles.

Decrease of the Rest Latitudes of the Large Scale Unipolar Regions Figure 7 shows that the so-called rest latitudes of the neutral lines between the large scale unipolar regions decreased gradually over the past 11 Schwabe cycles. The lower one of the rest latitudes gradually approached the solar equator during the 20th century Grand Maximum, leaving a small band of unipolar region growing from around the equator to the (lower) rest latitude. This is another signal of the Transition. This may be an indication of a gradual evolution of the meridional circulation, which may, in turn, be related to some kind of relatively longperiod oscillation of the tachocline.

Remarkable Delays in the Aftermath of Solar Cycle \#23 That cycle reached its maximum sunspot number in September 2001. Although the subsequent sunspot minimum would be expected to occur somewhere in 2005 or 2006, this did not happen. It came in 2008.5 (Fig. 2a) while polar maximum was attained in about 2009.5, which is 9 years after the maximum of cycle 22 . This may be related to changes in the meridional flow.

\section{A Pulsating Tachocline?}

In this final Section we introduce the hypothesis that the tachocline undergoes systematic motions with relatively small amplitudes, such that its equatorial parts show a component that moves periodically up- and downward. These motions could be called pulsations but they might, upon further research, appear to have another character.

The magnetic loops that will appear as sunspots at the photospheric level, have detached themselves from the tachocline when the latters field strength had grown to $6 \times 10^{4}$ Gauss, a value that is the average of the various determinations that have so far been published, as described earlier in this paper. We add at this point that this value of $6 \times 10^{4}$ Gauss is not critical for the discussion below, as will appear also from the following:

While rising, the loops expand and their field strengths decrease, in relation to the (decreasing) surrounding gas density; this causes the loops to expand. The deeper the tachocline source is situated, the smaller is therefore the resulting sunspot's photospheric magnetic field. Hence, the actual problem is that of the relation between the depth variation and related amplitude of the pulsating tachocline and the observed photospheric sunspot field strengths.

Assume an average ('standard') tachocline depth $z(0)$, where $z$ is the vertical ordinate. We place $z=0$ at this assumed 'standard' tachocline depth. The consequent umbral magnetic field corresponding with the standard tachocline depth will be called $B_{0}$. We now assume that the tachocline moves up or down, above or below the standard level. When the 
tachocline is situated at another depth than the standard value the consequent umbral magnetic field also assumes another value, that we will call $B_{\text {phot }}$. Hence the ratio between the observed photospheric field strength $B_{p h o t}$ and the standard strength $B_{o}$ is

$$
B_{\text {phot }} / B_{0}=\{P(0) / P(z)\}^{1 / 2} \text {, }
$$

where $P$ is the gas pressure.

The next problem is that of the relation between the depth of the tachocline and the value of $B_{\text {phot }}$, in short that of the distance between the standard tachocline depth and the actual depth, in relation to the photospheric values. In still other words: we want to find the semi-amplitude of the assumed pulsation.

For the solar interior at average tachocline depths $(\sim 200,000 \mathrm{~km})$ we have (Allen 1976)

$$
d \log P(z) / d z=0.0091 \text { per } 1000 \mathrm{~km} .
$$

Combining Eqs. (2) and (3) yields

$$
\text { Semi-amplitude }=\left(2 \log \left(B_{\text {phot }} / B_{0}\right) / 0.0091\right) \times 1000 \mathrm{~km} .
$$

The various components of Fig. 4 show that in the period between 1960 and 2000 the average umbral magnetic field had an average strength of $\sim 2450 \mathrm{G}$ (cf. Sect. 4) with a weakly defined semi-amplitude of some $300 \mathrm{G}$. According to Eq. (4) this implies a tachocline pulsation with a semi-amplitude of $\sim 12,000 \mathrm{~km}$. The full pulsation, twice this value, may be compared with the tachocline thickness, about 25,000 to $30,000 \mathrm{~km}$. Hence, we are dealing with a fairly small full amplitude, still inside the average ('standard') thickness of the tachocline.

With regard to the Transition we find that a decrease of the sunspot field from 2500 to 2000 Gauss, as happened between the years 2000 and 2010 (cf. Figs. 4a and 4b), corresponds, according to Eq. (4), with a semi-amplitude of 21,000 km. Hence, the recent Transition can be explained by a downward pulsation of the tachocline over that distance. Again: a distance smaller than the thickness of the tachocline. The small amplitude of the pulsations, where the tachocline remains in broad outline within its boundaries may explain why this hypothetical pulsation has not been detected by helioseismic techniques. Yet, Antia and Basu (2011) find from their helioseismic analysis that some parameters do show changes with time, while Basu et al. (2012) mention the thinning of the relevant layers during solar cycle 23 .

Pulsations? The question is relevant whether the tachocline is really 'pulsating'. Another viewpoint may be that the tachocline, as observed by helioseismology, is undergoing a differential rotation with respect to the solar core that leads to torsional oscillation of the whole convective layer (cf. Charbonneau 2010). In that line, Duhau and De Jager (2010) wrote "... we conclude that torsional oscillations stabilize the tachocline-convective layer system motions, leading to transfer of angular momentum in the latitudinal direction from the core spin to the tachocline..." Flux transport dynamo models (for a review see Dikpati and Gilman 2009) seem to contain all the necessary ingredients for realistic simulations of solar dynamo action. However, they predict a strong sunspot cycle 24 (Dikpati et al. 2006), comparable with those that occurred in the recent Grand Maximum. Observations instead indicate that this episode has ended (see Duhau and de Jager 2010). While these models assume a constant poloidal source at the tachocline-convective layer boundary these recent results indicate that the failure of that model is due to the fact that the conditions at the tachoclineconvective layer boundary suddenly changed during the transition(s), a possibility that is not incorporated in the flux transport models. These changing boundary conditions during 
the chaotic transitions are likely due to a sudden change of orientation of the tachoclineconvective motions with respect to the relic field seated at the core. In fact, for starting the dynamo loop, flux transport dynamo models need a seed field at the tachocline-convective layer boundary. Also, a relic field appears to be essentially necessary for having the radiative interior rotating as a rigid body and evidences indicating that his field is pointing southward have been provided since Cowling (1945) suggested its existence (reviews in Mursula et al. 2001, Duhau and de Jager 2010). The persistence of a negative polar field component at the transition, as found here, gives further support to these findings.

Non-linear Pulsations? These foregoing considerations seem to support the hypothesis that the tachocline may present motions of different kind that could be interpreted as pulsations with a small, hardly detectable amplitude. The most striking component is the pulsation of the equatorial diameter with an eleven years period and with a semi-amplitude of $12,000 \mathrm{~km}\left(0.017 R_{0}\right)$. Another conclusion is that the Transition corresponds to a downward displacement of the tachocline over $21,000 \mathrm{~km}$.

There is also evidence that there must be more periodicities acting than this sole one. This evidence is given by the four additional major periodicities: the two Gleissberg periods (the short and the long one), the Suess and the Hallstatt periodicities. Their analysis can be used to yield information on the variation of the dynamo (cf. also Fig. 13): During the negative phases of the Hallstatt periodicity of some 2000 years, Grand Minima cluster together (Steinhilber et al. 2010). They do not occur during the positive phase. It is known that the minimum umbral field strength that is needed for a spot to be visible as such, is $\sim 1500 \mathrm{G}$. In the very extreme case of a hypothetical Grand Minimum with zero sunspots, the umbral field strength must, hence, be smaller than or at most equal to $1500 \mathrm{G}$. That would be the case for a downward movement of the tachocline over $45,000 \mathrm{~km}\left(0.06 R_{0}\right)$. It seems probable that this fairly large shift of the tachocline depth is the reason why such extreme Maunder-type minima have not yet been observed.

Origin of Pulsations The origin of the tachocline 'pulsations' could most probably be found in the frame of the meridional circulation because the physical conditions in the tachocline area are at least partly related to the meridional circulation. By this circulation, magnetic flux and angular momentum are transported. The circulation consists of large cells that show up near the surface as an equatorward flow of $\sim 20 \mathrm{~m} / \mathrm{s}$. The return flow, deeper inside, is some ten times slower. The total circulation time is $\sim 10$ years. The situation that Grand Minima occur solely during negative phases of the Hallstatt oscillation may be explained by the assumption that the Hallstatt oscillation is due to a change of symmetry of the tachocline-convective motions with respect to the relic field seated at the core.

But the situation may be more complicated. Noteworthy is the finding of a double (or even triple) cell structure (Haber et al. 2002; Zhao et al. 2013). Haber et al. found that the equatorward streaming covers depths between 0.83 and $0.92 R_{\text {sun }}$ and the poleward one between $0.75-0.83 R_{\text {sun }}$ and $0.92-1.0 R_{\text {sun }}$. These boundaries are no fixed quantities, they change pseudo-randomly in the course of the solar cycle. Variation of the flow during the Transition are noteworthy: during solar cycle 23 the meridional flow extended up to the poles while in cycle 22 the circulation had switched equatorward near latitude $60^{\circ}$ (Dikpati et al. 2010). The authors suggested that this may have been at the origin of the longer duration of cycle 23. Nandy et al. (2011) introduced a fast meridional flow in the first half of the solar polar cycle followed by a slower flow in the second half. It appears that this introduction is able to reproduce the main characteristics of the sunspot cycle 23. Zhao et al. (2013) even find indications that the meridional flow does not extend as deep as to the bottom of the 
convection zone. Various computer simulations by other authors arrive at similar results: the velocity of the meridional circulation and its changes seem an essential aspect for explaining the Transition. More stochastic phenomena may occur too: a case is described in which a submerged cell, originating in the northern hemisphere disrupts the poleward flow and the symmetry in the streaming (Haber et al. 2002).

For understanding the assumed tachocline 'pulsations', we note that the radial distance of the tachocline to the solar center depends, among other things, on the amount of downward overshoot from the convective region. A sheer layer of radial differential rotation defines the conditions at the bottom of the convection zone (Choudhuri et al. 1995). This limits the meridional circulation to a lower bound of $\sim 0.7 R_{\text {sun }}$ (Gilman and Miesch 2004) and we suggest that this, in turn, influences or even determines the lowest position that the tachocline can take and the consequent umbral field strength. The physical origin of the tachocline pulsations may be sought in this direction. Numerical model calculations (Sule et al. 2005) indeed show how the meridional circulation changes the shape and position of the tachocline. Near the pole the tachocline is thinnest. This may find its origin in the counter-rotating meridional circulation that appears to develop in the polar regions (Rogers and MacGregor 2010, 2011).

It is obvious that the above considerations must be worked out and developed on base of an MHD analysis. A promising development that runs more or less parallel to this line of thought is found in the work of Mininni and Gomez (2002) and in that of Hiremath (2010). In the first quoted paper the MHD equations are developed and described. In the second paper, Hiremath (2010), mentions two possible ways of approach in understanding the solar dynamo: the turbulent dynamo and the MHD oscillatory mechanisms, and discusses the latter on basis of the two equations for an axisymmetric magnetic field structure in cylindrical geometry, as forwarded by Parker (1955). This theme has more recently been studied by Moss et al. (2008) and Usoskin et al. (2008). These equations couple the differential rotation to the poloidal and toroidal fields. Their application forecasts Alfvén waves that originate in the convective layer. They do explain the 22-years periodicity and it is obvious from the used equations, that there must be more such quasi-periodicities. It is not improbable that these are at the basis of the 'pulsations' of the tachocline.

The crucial question, yet to be studied, is if this MHD approach makes it possible to obtain quantitative agreement with the postulated up- and downward motions of the tachocline. Answering this question goes beyond the present review but is an obvious follow-up.

Acknowledgements This publication, a combination of a review and of a research paper is based on the work of many colleagues from whom we have collected information, quoted statements and copied diagrams. They have been mentioned in the text. Without all that, this paper could not have been written. Many sincere thanks, colleagues!

We are also deeply obliged to the two referees of this paper, who carefully and patiently have studied it and made a number of very useful quality-improving remarks.

In addition we mention Gleissberg. His cycle is one of the essential aspects of solar variability. One of the authors (CdeJ) met him for the first time in 1952 during the IAU General Assembly in Rome: a friendly and modest man. Less than ten years before he had introduced the Gleissberg cycle. Virtually no astrophysicist believed him: At that time the criticism was correct: the 'discovery' had been made on the basis of sunspot observations over only 2 centuries, hence including just slightly more than two of his 88 years 'periods'. According to current scientific standards such a correlation is insignificant and modern referees would have suggested not to publish his paper. But the scientific intuition of the prominent astrophysicist Albrecht Unsöld, editor-in-chief and sole referee of Zeitschrift für Astrophysik, took the decision that in hindsight appears to be correct.

Open Access This article is distributed under the terms of the Creative Commons Attribution 4.0 International License (http://creativecommons.org/licenses/by/4.0/), which permits unrestricted use, distribution, and reproduction in any medium, provided you give appropriate credit to the original author(s) and the source, provide a link to the Creative Commons license, and indicate if changes were made. 


\section{References}

O. Adriani et al. (PAMELA collaboration), Time dependence of the proton flux measured by the PAMELA experiment during the last solar minimum: July 2006-December 2009. Astrophys. J. 765(91), 1-8 (2013).

S.-I. Akasofu, Single spots, unipolar magnetic regions and pairs of spots. Geophys. Res. Lett. 41, 3 (2014). doi:10.1002/2014GRL060319

S.-I. Akasofu, Single spots, unipolar magnetic regions and pairs of spots (II), the development of sunspot pairs and the Hale boundary. Geophys. Res. Lett. 42, 3698 (2015). doi:10.1002/2014GLD62887

C.W. Allen, Astrophysical Quantities, 3rd edn. (Athlone, London, 1976), p. 163

R. Ananthakrishnan, Prominence activity (1905-1952). Proc. Indian Acad. Sci. 40, 72 (1954)

H.M. Antia, S. Basu, Revisiting the solar tachocline; average properties and temporal variations. Astrophys. J. Lett. 735, L45 (2011). doi:10.1088/2041-8205/735/2/L45

A.J.P. Aparicio, J.M. Vaquero, M.C. Gallego, The proposed "Waldmeier discontinuity": how does it affect the sunspot cycle characteristics. J. Space Weather Space Clim. 2, A12 (2012)

O.P. Aslam, M. Badruddin, Solar modulation of cosmic rays during the declining and minimum phases of Solar Cycle 23: comparison with past three solar cycles. Sol. Phys. 279, 269-288 (2012)

H.W. Babcock, H.D. Babcock, The Sun's magnetic field, 1952-1954. Astrophys. J. 121, 349 (1955)

H.D. Babcock, W.C. Livingston, Changes in the sun's polar magnetic field. Science 127, 1058 (1958)

A. Balogh, H.S. Hudson, K. Petrovay, An introduction to the solar activity cycle. Overview of causes and consequences. Space Sci. Rev. 186, 1 (2014)

G.A. Basilevskaya, V.S. Makhmutov, A.I. Sladkova, Gnevyshev gap effects in solar energetic particle activity. Adv. Space Res. 38, 484-488 (2006)

S. Basu, Seismology of the base of the solar convection zone. Mon. Not. R. Astron. Soc. 288, 527 (1997)

S. Basu, J. Schou, Does the tachocline shows solar cycle related changes? Sol. Phys. 192, 481 (2000)

S. Basu, A.-M. Broomhall, W.J. Chaplin, Y. Elsworth, Thinning of the Sun's magnetic layer, the peculiar solar minimum could have been predicted. Astrophys. J. 758(1), 43 (2012)

J. Beer, K.G. McCracken, J. Abreu, U. Heikkilä, F. Steinhilber, Cosmogenic radionuclides as an extension of the Neutron Monitor Era into the past: potential and limitations. Space Sci. Rev. 176 (1-4), 89-100 (2013)

L. Bengtsson, S. Koumoutsaris, R.-M. Bonnet, R.P. Allan, C. Fröhlich, J. Heintzenberg, P. Ingmann, R. Kandel, N.G. Leb, B. Soden, K.E. Trenberth, Observing and modeling Earth's energy flows. Surv. Geophys. 33, 333-816 (2012)

J.D. Bohlin, Extreme ultraviolet observations of coronal holes, I: location, sizes and evolution. Sol. Phys. 51, 377 (1977)

T. Borrmann, H. Fichtner, On the dynamics of the heliosphere on intermediate and long time-scales. Adv. Space Res. 35, 2091-2101 (2005)

A. Brandenburg, D. Sokoloff, K. Subramanian, Current status of turbulent dynamo theory. Space Sci. Rev. 169, 123-157 (2012)

A.-M. Broomhall, W.J. Chaplin, Y. Elsworth, S.T. Fletcher, R. New, Is the current lack of solar activity only skin deep? Astrophys. J. 700, L162-L165 (2009)

A.S. Brun, J. Toomre, Turbulent convection under the influence of rotation. Astrophys. J. 570, 865 (2002)

V. Bumba, R. Howard, Large-scale distribution of solar magnetic fields. Astrophys. J. 141, 1502 (1965)

P. Caligari, F. Moreno-Insertis, M. Schussler, Emerging flux tubes in the solar convection zone, I: asymmetry, tilt and emergence latitude. Astrophys. J. 441, 886 (1995)

P.S. Cally, Three-dimensional magneto-shear instabilities in the solar tachocline. Mon. Not. R. Astron. Soc. 339, 957 (2003)

R. Cameron, M. Schüssler, The crucial role of surface magnetic fields for the solar dynamo. Science $\mathbf{3 4 7}$, 1333 (2015)

P. Charbonneau, Living reviews of solar physics (2010). http://www.livingreviews.org/lrsp-2010-3

P. Charbonneau, J. Christensen-Dalsgaard, R. Henning, R.M. Larsen, J. Schou, M.J. Thompson, Helioseismic constraints on the structure of the solar tachocline. Astrophys. J. 527, 445-460 (1999). doi:10.1086/308050

P. Charbonneau, G. Blais-Laurier, C. St-Jean, Intermittence and phase persistence in a Babcock-Leighton model of the solar cycle. Astrophys. J. 616, L183 (2004)

A.R. Choudhuri, M. Schüssler, M. Dikpati, The solar dynamo with meridional circulation. Astron. Astrophys. 303, L29 (1995)

A.R. Choudhuri, P. Chatterjee, J. Jiang, Predicting solar cycle 24 with a solar dynamo model. Phys. Rev. Lett. 98, 131103 (2007)

J. Christensen-Dalsgaard, An overview of helio- and astero-seismology, in Challenge to the Theories of the Structure of Moderate Mass Stars, ed. by D. Gough, J. Toomre (1991), p. 388 
F. Clette, L. Svalgaard, J.M. Vaquero, E.W. Cliver, Revisiting the sunspot number. A 400 year perspective on the solar cycle. Space Sci. Rev. 186, 35 (2014)

F. Clette, E.W. Cliver, L. Lefèvre, L. Svalgaard, J.M. Vaquero, Revision of the sunspot number. Space Weather 13, 529 (2015)

M.A. Clilverd, E. Clarke, H. Rishbeth, T. Ulrich, T.G.D. Clark, Solar activity levels in 2001. Astron. Geophys. 44, 5.21-24 (2003)

M.A. Clilverd, E. Clarke, T. Ulich, H. Rishbeth, M.J. Jarvis, Predicting solar cycle 24 and beyond. Space Weather 4, S09005 (2006). doi:10.1029/2005SW000207

E.W. Cliver, The extended cycle of solar activity and the sun's 22 year magnetic cycle. Space Sci. Rev. 186, 169 (2014)

E.W. Cliver, A.G. Ling, 22 year patterns in the relationship of sunspot number and tilt angle of cosmic ray intensity. Astrophys. J. 551(2), 189 (2001a)

E.W. Cliver, A.G. Ling, Coronal mass ejections, open magnetic flux, and cosmic-ray modulation. Astrophys. J. 556(1), 432-437 (2001b)

E.W. Cliver, A.G. Ling, The floor in the solar wind magnetic field revisited. Sol. Phys. 274, 285-301 (2011)

T.G. Cowling, On the suns general magnetic field. Mon. Not. R. Astron. Soc. 105, 166 (1945)

C. de Jager, Structure and dynamics of the solar atmosphere, in Encyclopaedia of Physics, vol. 52 (Springer, Berlin, 1959), pp. 80-362

C. de Jager, Solar forcing of climate, I: solar variability. Space Sci. Rev. 120, 197 (2005)

C. de Jager, Solar forcing of climate. Surv. Geophys. 33, 445-451 (2012)

C. de Jager, S. Duhau, The variable solar dynamo and the forecast of solar activity; influence on terrestrial surface temperature, in Global Warming in the 21th Century, ed. by J.M. Cossia (Nova, Hauppauge, 2011). ISBN 978-1-61728-980-4, Chap. 3

C. de Jager, S. Duhau, Sudden transitions and grand variations in the solar dynamo, past and future. J. Space Weather Space Clim. 2, A07 (2012). doi:10.1051/swsc/2012008

C. de Jager, H. Nieuwenhuijzen, Terrestrial ground temperature variations in relation to solar magnetic variability, including the present Schwabe cycle. Nat. Sci. 5, 1112-1120 (2013)

C. de Jager, I.G. Usoskin, On possible drivers of sun-induced climate change. J. Atmos. Sol.-Terr. Phys. 68, 2053 (2006)

C. de Jager, S. Duhau, B. van Geel, Quantifying and specifying the solar influence on terrestrial surface temperature. J. Atmos. Sol.-Terr. Phys. 72, 926 (2010)

M. Dikpati, The importance of the solar tachocline. Adv. Space Res. 35, 322 (2005)

M. Dikpati, P.A. Gilman, Flux-transport solar dynamos. Space Sci. Rev. 144, 67 (2009)

M. Dikpati, G. de Toma, P.A. Gilman, Predicting the strength of solar cycle 24 using a flux-transport dynamobase tool. Geophys. Res. Lett. 33, L05102 (2006)

M. Dikpati, P.A. Gilman, G. de Toma, G.K. Ulrich, Impact of changes in the Sun's conveyor belt on recent solar cycles. Geophys. Res. Lett. 37, L1417 (2010). 2010. doi:10.1029/2010GL044143

S. D’Silva, A.R. Choudhuri, Astron. Astrophys. 272, 621 (1993)

S. Duhau, An early prediction of maximum sunspot number in solar cycle 24. Sol. Phys. 213, 203 (2003)

S. Duhau, Y. Chen, The sudden increase of solar geomagnetic activity after 1923. Geophys. Res. Lett. 29, 1628 (2002). doi:10.1029/2001GL013953

S. Duhau, C. de Jager, The solar dynamo and its phase transitions during the last millennium. Sol. Phys. 250, $1(2008)$

S. Duhau, C. de Jager, The forthcoming grand minimum of solar activity. J. Cosmol. 8, 1983-1999 (2010)

J.R. Elliot, D.O. Gough, Calibration of the thickness of the tachocline. Astrophys. J. 516, 475 (1999)

J. Feynman, A. Ruzmaikin, The centennial Gleissberg cycle and its association with extended minima. J. Geophys. Res. 119, 6027-6041 (2014)

G.H. Fisher, Y. Fan, D.W. Longcope, M.G. Linton, A. Pevtsov, The solar dynamo and emerging flux. Sol. Phys. 192, 119 (2000)

L. Fletcher, P.J. Cargill, S.K. Antiochos, B.V. Gudiksen, Structure in the outer solar atmosphere. Space Sci. Rev. 188, 211-249 (2015). doi:10.1007/s11214-014-0111-1

P.A. Gilman, M.S. Miesch, Limits to penetration of meridional circulation below the solar convection zone. Astrophys. J. 611, 568 (2004)

R. Giovanelli, Solar magnetism. Sol. Phys. 80, 21 (1982)

W. Grotrian, H. Künzel, Über den Induktionsflusz durch die Sonnenflecken. Z. Astrophys. 28, 28 (1950)

D.A. Haber, B.W. Hindman, J. Toomre, R.S. Bogart, R.M. Larsen, F. Hill, Evolving submerged meridional circulation cells within the upper convection zone revealed by ring-diagram analysis. Astrophys. J. 570, 855 (2002)

D.H. Hathaway, The solar cycle, in Living Reviews in Solar Physics (2010). http://www.livingreviews.org/ 1rsp-2010-1 
D.H. Hathaway, R.M. Wilson, J. Reichmann, A synthesis of solar cycle prediction. J. Geophys. Res. 104, 22375 (1999)

D.H. Hathaway, R.M. Wilson, E.J. Reichmann, Group Sunspot Numbers, sunspot cycle characteristics. Sol. Phys. 211, 357 (2002)

B. Heber, A. Kopp, J. Gieseler, R. Müller-Mellin, H. Fichtner, K. Scherer et al., Modulation of galactic cosmic ray protons and electrons during an unusual solar minimum. Astrophys. J. 699, 1956 (2009)

K.M. Hiremath, Physics of the solar cycle, new views. Sun Geosph. 5(1), 17 (2010)

J.T. Hoeksema, in Solar and Stellar Variability; Impact on Earth and Planets, ed. by A.G. Kosovichev, A.H. Andrei, J.P. Rozelot. IAU Symp., vol. 264 (2010), p. 222

D.V. Hoyt, K.H. Schatten, Group Sunspot Numbers, a new solar activity reconstruction. Sol. Phys. 181, 491 (1998)

S. Ilonides, J. Zhao, A. Kosovichev, Detection of emerging sunspot regions in the solar interior. Science 333, 993-996 (2011)

J. Jiang, P. Chatterjee, A.R. Choudhuri, Solar activity forecast with a dynamo model. Mon. Not. R. Astron. Soc. 381, 1527 (2007)

R.P. Kane, Hysteresis of cosmic rays with respect to sunspot numbers during the recent sunspot minimum. Sol. Phys. 269, 451-454 (2011)

P.J. Käpylä, M.J. Korpi, I. Tuominen, Solar dynamo models with alpha-effect and turbulent pumping from local 3D convection calculations. Astron. Nachr. 327(9), 884-894 (2006)

M. Küker, G. Rüdiger, M. Schulz, Circulation-dominated solar shell dynamo models with positive alpha effect. Astron. Astrophys. 374, 301-308 (2001)

D.A. Lamb, C.E. DeForest, H.J. Hagenaar, C.E. Parnell, B.T. Welsch, Solar magnetic tracking II. The apparent unipolar origin of the quiet-sun flux. Astrophys. J. 674, 520-529 (2008)

C.O. Lee, J.G. Luhmann, J.T. Hoeksema, X. Sun, C.N. Arge, I. de Pater, Coronal field opens at lower height during the solar cycles 22 and 23 minimum periods: IMF comparison suggests the source surface should be lowered. Sol. Phys. 269, 367 (2011)

R.B. Leighton, Transport of magnetic fields in the sun. Astrophys. J. 140, 1547 (1964)

W. Livingston, F. Watson, A new solar signal: average sunspot magnetic field independent of activity cycle. Geophys. Res. Lett. 42, 9185 (2015)

W. Livingston, M.J. Penn, L. Svalgaard, Decreasing sunspot magnetic fields may explain unique $10.7 \mathrm{~cm}$ radio flux. Astrophys. J. Lett. 757, L8 (2012). doi:10.1088/2041-8205/757/1/L8

M. Lockwood, Solar influences on global and regional climates. Surv. Geophys. 33, 503-534 (2012). doi:10.1007/s10712-012-9181-3

M. Lockwood, Reconstruction and prediction of variations in the open solar magnetic flux and interplanetary conditions (2013). http://livingreviews.org/lrsp-2013-4

M. Lockwood, A.P. Rouillard, I.D. Finch, The rise and fall of the open solar flux during the current Grand solar Maximum. Astrophys. J. 700, 637-939 (2009). doi:10.1088/0004-637X/700/2/937

M. Lockwood, R.G. Harrison, T. Woollings, S.K. Solanki, Are cold winters in Europe associated with low solar activity? Environ. Res. Lett. 5, 024001 (2010). doi:10.1088/1748-9326/2/024001

V.I. Makarov, A.G. Tlatov, D.K. Callebaut, Secular and cyclic variation of polar activities on the sun, in Multi Wavelength Investigations of Solar Activity, ed. by A.V. Stepanov, E.E. Benevolenskaya, A.G. Kosovichev. Proc. IAU Symposium, vol. 229 (2004), p. 49

P.N. Mayaud, The $a a$ index, a 110 years series characterizing the magnetic activity. J. Geophys. Res. 67, 6870 (1972)

K.G. McCracken, J. Beer, F. Steinhilber, J. Abreu, A phenomenological study of the cosmic ray variations over the past 9400 years, and their implications regarding solar activity and the solar dynamo. Sol. Phys. 286, 609-627 (2013)

F.B. McDonald, W.R. Webber, D.V. Reames, The unusual time histories of galactic and anomalous cosmic rays at $1 \mathrm{AU}$ over the deep solar minimum of 23/24. Geophys. Res. Lett. 37, L18101 (2010)

P.S. McIntosh, in The Physics of Sunspots, vol. 7, ed. by L.E. Crom, J.H. Thomas, Sacramento Peak (1981), pp. 7-54

P.S. McIntosh, P.R. Wilson, A new model for flux emergence and the evolution of sunspots and the large-scale field. Sol. Phys. 97, 59-79 (1985)

R.A. Mewaldt, Cosmic rays in the heliosphere: requirements for future observations. Space Sci. Rev. 1(76), 1-4 (2013). 365-390

R.A. Mewaldt, A.J. Davis, K.A. Lave, R.A. Leske, E.C. Stone, M.E. Wiedenbeck et al., Record-setting cosmic-ray intensities in 2009 and 2010. Astrophys. J. Lett. 273, L1 (2010)

P.D. Mininni, D.O. Gomez, Study of stochastic fluctuations in a shell dynamo. Astrophys. J. 573, 454 (2002)

D. Moss, D. Sokoloff, I. Usoskin, V. Tutubalin, Solar grand minima and random fluctuations in dynamo parameters. Sol. Phys. 250, 221 (2008) 
A. Muñoz-Jaramillo, N.R. Sheeley Jr., J. Zhang, E.E. DeLuca, Calibrating 100 years of polar faculae measurements: implications for the evolution of the heliospheric magnetic field. Astrophys. J. 753, 146 (2012)

A. Muñoz-Jaramillo, L.A. Balmaceda, E.E. DeLuca, Using the dipolar quadrupole moments to improve solarcycle predictions based on polar magnetic fields. Phys. Rev. Lett. 111, 041106 (2013a)

A. Muñoz-Jaramillo, P.C.H. Martens, D. Nandy, Helioseismic properties of the solar dynamo. ASP Conf. Ser. 478, 271 (2013b)

A. Muñoz-Jaramillo, R.R. Senkpeil, D.W. Longcope, A.G. Tlatov, A.A. Pevtsov, L.A. Balmaceda, E.E. DeLuca, P.C.H. Martens, The minimum of solar cycle 23: as deep as it could be? Astrophys. J. 804, $68(2015)$

K. Mursula, I.G. Usoskin, G.A. Kovaltsov, Persistent 22-year cycle in sunspot activity: Evidence for a relic solar magnetic field. Sol. Phys. 198, 51 (2001)

R. Muscheler, J. Beer, M. Vonmoos, Causes and timing of the $8200 \mathrm{BP}$ event inferred from the comparison of the GRIP [ ${ }_{\text {loc }}=$ pre]10Be and the tree ring [ $l o c=$ pre]14C record. Quat. Sci. Rev. 23, 2101-2111 (2004)

Yu.A. Nagovitsyn, To the description of long-term variations in the solar magnetic flux; the sunspot area index. Astron. Lett. 31(8), 557 (2005)

Yu.A. Nagovitsyn, A.A. Pevtsov, W.C. Livingston, On a possible explanation of the long-term decrease in sunspot field strength. Astrophys. J. Lett. 758, L20 (2012)

Y. Nakagawa, A numerical study of the solar cycle, in Solar Magnetic Fields, ed. by R. Howard (Reidel, Dordrecht, 1971), pp. 725-736

D. Nandy, A. Muñoz-Jaramillo, P.C.H. Martens, The unusual minimum of sunspot cycle 23 caused by meridional plasma flow variations. Nature 471, 80 (2011).

H. Nevalinna, E. Kataja, An extension of the geomagnetic activity index series aa for two solar cycles (18441868). Geophys. Res. Lett. 20, 2700 (1993)

M. Ossendrijver, The solar dynamo. Astron. Astrophys. Rev. 11, 287 (2003)

E.N. Parker, Hydromagnetic dynamo models. Astrophys. J. 122, 293 (1955)

M.J. Penn, W.C. Livingston, Temporal changes in sunspot umbral magnetic fields and temperatures. Astrophys. J. Lett. 649, L45 (2006)

M.J. Penn, W.C. Livingston, Are sunspots different during this solar minimum? Eos 90, 257 (2009)

G.J.D. Petrie, Solar magnetism in the polar regions. Living Rev. Sol. Phys. 12, 5 (2015). doi:10.1007/ lrsp-2015-5, http://www.livingreviews.org/lrsp-2015-5

A.A. Pevtsov, W.C. Livingston, Yu.A. Nagovitsyn, On a possible explanation of the long-term decrease in sunspot field strength. Astrophys. J. Lett. 758, L10 (2012)

M.S. Potgieter, Time-dependent cosmic-ray modulation: role of drifts and interaction regions. Adv. Space Res. 13, 239-249 (1993)

M.S. Potgieter, Solar cycle variations and cosmic rays. J. Atmos. Sol.-Terr. Phys. 70, 207-218 (2008)

M.S. Potgieter, Solar modulation of cosmic rays. Living Rev. Sol. Phys. 10, 3-66 (2013)

M.S. Potgieter, The charge-sign dependent effect in the solar modulation of cosmic rays. Adv. Space Res. 53, 1415-1425 (2014)

M.S. Potgieter, E.E. Vos, M. Boezio, N. de Simone, V. di Felice, V. Formato, Modulation of galactic protons in the heliosphere during the unusual solar minimum from 2006 to 2009: a modeling approach. Sol. Phys. 289, 391-406 (2014)

R. Rezaie, C. Beck, M. Collados, J.M. Borrero, A. Lag, W. Schmidt, Variation of sunspot properties between 1999 and 2014. Astron. Astrophys. 578, A43 (2015)

R.S. Richardson, M. Schwarzschild, in Problemi della Fisica Solare, Atti del Convegno, 14-19 Settembre 1952. Atti dei Convegni Fondazione A. Volta, vol. 11 (Accad. Naz. dei Lincei, Rome, 1953), pp. 228249

T.M. Rogers, K.B. MacGregor, On the interaction of internal gravity waves with a magnetic field, I: artificial wave forces. Mon. Not. R. Astron. Soc. 401, 191 (2010)

T.M. Rogers, K.B. MacGregor, On the interaction of internal gravity waves with a magnetic field, II: convective forces. Mon. Not. R. Astron. Soc. 410, 946 (2011)

G. Rüdiger, Solar dynamo. Scolarpedia 3(1), 3444 (2008)

C.T. Russell, On the possibility of deducing interplanetary and solar parameters from geomagnetic records. Sol. Phys. 42, 259 (1975)

C.T. Russell, T. Mulligan, The 22-year variation of geomagnetic activity: implications for the polar magnetic field of the Sun. Geophys. Res. Lett. 22, 3287 (1995). doi:10.1029/95GL03086

C.T. Russell, J.G. Luhman, L.K. Jian, How unprecedented a solar minimum? Rev. Geophys. 48(2), RG2004 (2010). doi:10.1029/2009RG000316

A. Ruzmaikin, Clustering of emerging magnetic flux. Sol. Phys. 181, 1 (1998)

A. Savcheva, J.W. Cirtain, E.E. DeLuca, L. Golub, Does a polar coronal hole flux emergence follow a Halelike law? Astrophys. J. Lett. 702, L32 (2009). doi:10.1088/0004-637X/702/1/L32 
K.H. Schatten, P.H. Scherrer, L. Svalgaard, J.M. Wilcox, Using dynamo theory to predict the sunspot number. Geophys. Res. Lett. 5, 411-414 (1978)

K. Scherer, H. Fichtner, T. Borrmann, J. Beer, L. Desorgher, H.-J. Fahr, S.E.S. Ferreira, U.W. Langner, M.S. Potgieter, B. Heber, J. Masarik, N. Shaviv, J. Veizer, Interstellar-terrestrial relations: variable cosmic environments, the dynamic heliosphere, and their imprints on terrestrial archives and climate. Space Sci. Rev. 127, 327-465 (2006)

B. Schmieder, V. Archontis, V. Pariat, Magnetic flux emergence along the solar cycle. Space Sci. Rev. 186, 227-250 (2014). doi:10.1007/s11214-014-0088-9

D.J. Schove, The sunspot cycle, 649 BC to AD 2000. J. Geophys. Res. 60(2), 127 (1955)

N.R. Sheeley Jr., Measurements of solar magnetic fields. Astrophys. J. 144, 723 (1966)

N.R. Sheeley Jr., Polar faculae during the interval 1906-1975. J. Geophys. Res. 81, 3462 (1976)

N.R. Sheeley Jr., Polar faculae, 1906-1990. Astrophys. J. 374, 386 (1991)

N.R. Sheeley Jr., A century of polar faculae variations. Astrophys. J. 680, 1553 (2008)

J.A. Simpson, H.W. Babcock, H.D. Babcock, Association of unipolar magnetic region on the sun with changes of primary cosmic ray intensity. Phys. Rev. 98, 1402 (1955)

S.K. Solanki, Sunspots, an overview. Astron. Astrophys. Rev. 11, 153 (2003)

S.K. Solanki, N.A. Krivova, Analyzing solar cycles. Science 334, 916 (2011)

S.K. Solanki, M. Schüssler, M. Fligge, Evolution of the sun's large-scale magnetic field since the Maunder minimum. Nature 208, 445-447 (2000)

S.K. Solanki, I.G. Usoskin, B. Kromer, M. Schüssler, J. Beer, Unusual activity of the sun during recent decades compared to the previous 11.000 years. Nature 431, 1084 (2004)

F. Steinhilber, J. Beer, C. Fröhlich, Total solar irradiance during the holocene. Geophys. Res. Lett. 36, 19704 (2009). doi:10.1029/2009GL040142

F. Steinhilber, J.A. Abreu, J. Beer, K.G. McCracken, Interplanetary magnetic field during the past 9300 years inferred from cosmogenic radionuclides. J. Geophys. Res. 115, A01104 (2010). doi:10.1029/2009JA014193

R.D. Strauss, M.S. Potgieter, Is the highest cosmic rays yet to come? Sol. Phys. 289(8), 3197-3205 (2014)

A. Sule, G. Rüdiger, R. Arlt, A numerical MHD model for the solar tachocline with meridional flow. Astron. Astrophys. 437, 11061 (2005)

L. Svalgaard, E.W. Cliver, A floor in the solar wind magnetic field. Astrophys. J. Lett. 661, L203 (2007)

L. Svalgaard, K.H. Schatten, Reconstruction of the sunspot group number: the backbone method. Sol. Phys. (2016). doi:10.1007/s111207-015-0815-8

L. Svalgaard, J.M. Wilcox, The Hale solar sector boundary. Sol. Phys. 49, 177-185 (1976)

L. Svalgaard, E.W. Cliver, Y. Kamide, Sunspot cycle 24. Smallest cycle in 100 years? Geophys. Res. Lett. 32, L01104 (2005)

S.M. Tobias, Modulation of solar and stellar dynamos. Astron. Nachr. 323, 417 (2002)

S.M. Tobias, F. Cattaneo, Shear-driven dynamo waves at high magnetic Reynolds number. Nature 497, 463465 (2013)

J. Tuominen, The latitude drift of sunspots. Z. Astrophys. 37, 145 (1954)

I.G. Usoskin, A history of solar activity over millennia. Living Rev. Sol. Phys. 10, 1 (2013)

I.G. Usoskin, K. Mursula, Long-term solar cycle evolution. Review of recent developments. Sol. Phys. 218, 319 (2003)

I.G. Usoskin, D. Sokoloff, D. Moss, Grand minima of solar activity and the mean-field dynamo. Sol. Phys. 254, 345 (2008). doi:10.1007/s11207-008-9293-6

I.G. Usoskin, G. Hulot, Y. Gallet, R. Roth, A. Licht, F. Joos, G.A. Kovaltsov, E. Thebault, A. Khokhlov, Evidence for distinct modes of solar activity. Astron. Astrophys. 562, L10 (2014)

G.M. Versteegh, Solar forcing of climate. 2. Evidence from the past. Space Sci. Rev. 120, 243 (2005)

A. Vourlidas, R.A. Howard, E. Esfandiari, S. Patsourakos, S. Tashiro, G. Michalek, Comprehensive analysis of coronal mass ejections; mass and energy properties over a full solar cycle. Astrophys. J. 722, 15221538 (2010)

M. Waldmeier, Die polare Protuberanzenzone. Z. Astrophys. 42, 34 (1957)

F.T. Watson, L. Fletcher, S. Dalla, S. Marshall, The long-term trend in magnetic fields of sunspot umbrae. Sol. Phys. 260, 5 (2009)

F.T. Watson, M.J. Penn, W. Livingston, A multi-instrument analysis of sunspot umbrae. Astrophys. J. 787, 22 (2014)

M.A. Weber, Y. Fan, Effects of radiative diffusion on thin flux tubes in solar-like convection. Sol. Phys. 290, 1295 (2015)

M.A. Weber, Y. Fan, M.S. Miesch, Comparing simulations of rising flux tubes through the solar convection zone with observations of solar active regions: constraining the dynamo filed strength. Sol. Phys. 287, 239 (2013) 
N.O. Weiss, Is the solar cycle an example of deterministic chaos? in Secular Solar and Geomagnetic Variations in the Last 10,000 Years, ed. by F.R. Stephenson, A.W. Wolvendale (Kluwer Academic, Dordrecht, 1988), pp. 69-77

N.O. Weiss, Modulation of the sunspot cycle. Astron. Geophys. 51, 3.9-13 (2010)

N.O. Weiss, M.J. Thompson, The solar dynamo. Space Sci. Rev. 144, 53-66 (2009)

N.O. Weiss, F. Cattaneo, C.A. Jones, Periodic and aperiodic dynamo waves. Geophys. Astrophys. Fluid Dyn. 30, 305-341 (1984)

K.L. Yeo, N.A. Krivova, S.K. Solanki, Solar cycle variation in solar irradiance. Space Sci. Rev. 186, 137 (2014)

K.L. Yeo, W.T. Ball, N. Krivova, S.K. Solanki, Y.C. Unruh, J. Morrell, UV solar irradiation in observations and NRLSSI and SATIRE-S modes. J. Geophys. Res. 120, A021277 (2015)

J. Zhao, R.S. Bogart, A.G. Kosovichev, T.L. Duvall, T. Hartlep, Detection of equatorward meridional flow and evidence of double-cell meridional circulation inside the sun. Astrophys. J. Lett. 774, L29 (2013)

C. Zwaan, Elements and patterns in the solar magnetic field. Annu. Rev. Astron. Astrophys. 25, 83-112 (1987) 